\title{
Fat composition in infant formula contributes to the severity of necrotising enterocolitis
}

\author{
Chhinder P. Sodhi ${ }^{1,2}$, William B. Fulton ${ }^{1,2}$, Misty Good ${ }^{3}$, Mustafa Vurma ${ }^{4}$, Tapas Das ${ }^{4}$, Chron-Si Lai ${ }^{4}$, \\ Hongpeng $\mathrm{Jia}^{1,2}$, Yukihiro Yamaguchi ${ }^{1,2}$, Peng $\mathrm{Lu}^{1,2}$, Thomas Prindle $\mathrm{Jr}^{1,2}$, John A. Ozolek ${ }^{5}$ and \\ David J. Hackam ${ }^{1,2 *}$ \\ ${ }^{1}$ Division of General Pediatric Surgery, Johns Hopkins University and Johns Hopkins Children's Center, Baltimore, \\ MD 21287, USA \\ ${ }^{2}$ Department of Surgery, Johns Hopkins University and Johns Hopkins Children's Center, Baltimore, MD 21287, USA \\ ${ }^{3}$ Department of Pediatrics, Division of Newborn Medicine, Washington University School of Medicine, St. Louis, \\ MO 63110, USA \\ ${ }^{4}$ Abbott Nutrition, a Division of Abbott Laboratories, Columbus, OH 43219, USA \\ ${ }^{5}$ Division of Pediatric Pathology and Department of Pathology, Children's Hospital of Pittsburgh, Pittsburgh, PA 15224, USA
}

(Submitted 21 February 2018 - Final revision received 22 May 2018 - Accepted 5 June 2018)

\section{Abstract}

Necrotising enterocolitis (NEC) is a devastating disease that typically affects formula-fed premature infants, suggesting that dietary components may influence disease pathogenesis. TAG are the major fat components of infant formula, and their digestion requires pancreatic lipases, which may be naturally deficient in premature neonates. We hypothesise that NEC develops partly from the accumulation of incompletely digested long-chain TAG-containing unsaturated fatty acids within the intestinal epithelial cells, leading to oxidative stress and enterocyte damage. We further hypothesise that the administration of a formula that contains reduced TAG ('pre-digested fat') that do not require lipase action may reduce NEC severity. To test these hypotheses, we induced NEC in neonatal mice using three different fat formulations, namely 'standard fat', 'pre-digested fat' or 'very low fat', and determined that mice fed 'standard fat' developed severe NEC, which was significantly reduced in mice fed 'pre-digested fat' or 'very low fat'. The expression level of the critical fat-digesting enzyme carboxyl ester lipase was significantly lower in the newborn compared with older pups, leading to impaired fat digestion. The accumulation of mal-digested fat resulted in the significant accumulation of fat droplets within the intestinal epithelium of the distal ileum, resulting in the generation of reactive oxygen species and intestinal inflammation. Strikingly, these changes were prevented in pups fed 'pre-digested fat' or 'very low fat' formulas. These findings suggest that nutritional formula containing a pre-digested fat system may overcome the natural lipase deficiency of the premature gut, and serve as a novel approach to prevent NEC.

\section{Key words: Necrotising enterocolitis: Inflammation: Infant nutrition: Reactive oxygen species}

Necrotising enterocolitis (NEC) is the leading cause of death from gastrointestinal disease in premature infants ${ }^{(1,2)}$. Although the pathogenesis of NEC remains incompletely understood, its development is nearly always seen after the administration of enteral feeds, suggesting that specific components of infant formula such as dietary fat may contribute to NEC pathogenesis ${ }^{(2)}$. In a rat model of experimental NEC, Caplan et $a l .{ }^{(3)}$ showed that the administration of PUFA arachidonic acid and DHA reduced the incidence of $\mathrm{NEC}^{(3)}$, whereas Lu et al. ${ }^{(4)}$ showed that PUFA reduced NEC severity partly through the reduction in expression of the lipopolysaccharide receptor, toll-like receptor $4\left(T_{L R} 4\right)^{(4)}$, supporting findings from our laboratory and others that TLR4 signalling plays a critical role in NEC pathogenesis ${ }^{(5-8)}$. These findings place the spotlight on how fat is handled in the premature intestine, and how failure of appropriate fat digestion may lead to NEC.

In the presence of the principal fat-digesting enzyme namely carboxyl ester lipase, ingested TAG are normally digested in the lumen of the duodenum and jejunum into NEFA and 2-monoacylglycerols, which are then emulsified into soluble micelles that are taken up by intestinal epithelial cells. In the intestinal epithelial cells, NEFA and 2-monoacylglycerols are then converted back to TAG. The TAG then combine with

Abbreviations: BSDL, bile-salt-dependent lipase; CEL, carboxyl ester lipase; DHE, dihydroethidium; MDA, malondialdehyde; NAC, $N$-acetylcysteine; NEC, necrotising enterocolitis; PDF, pre-digested fat; PFA, paraformaldehyde; qRT-PCR, quantitative real-time PCR; ROS, reactive oxygen species

* Corresponding author: D. J. Hackam, fax +1 4105025314 , email dhackam1@jhmi.edu 
cholesterol, protein and phospholipids to form chylomicrons that are transported out via lymph to the rest of the body ${ }^{(9)}$. Importantly, premature infants display a relative inability to digest and absorb TAG fat ${ }^{(10,11)}$ and may have underdeveloped capacities for chylomicron synthesis ${ }^{(12)}$. This potential inability of the immature intestine to adequately digest ingested fat could lead to the accumulation of TAG-containing unsaturated fatty acids in the ileum, where they would be at risk of becoming oxidised. The ensuing production of lipid peroxides could then induce oxidative stress and inflammation and contribute to the development of NEC.

We now seek to explore the role of fat digestion in the premature gut in greater detail in the pathogenesis of NEC. As described above, the critical enzyme required for fat digestion in the lumen of the intestine is carboxylic ester lipase (CEL) also called bile-salt-dependent lipase (BSDL) - which is secreted into the intestinal lumen by the exocrine pancreas $^{(13)}$. Exocrine pancreatic function is not fully developed at birth, and as a result CEL release is insufficient to adequately support fat absorption in the newborn ${ }^{(14,15)}$. In a remarkable demonstration of the nutritional synergy that exists between infant and mother, CEL is also secreted by the lactating mammary gland into the breast milk, and upon ingestion of breast milk the infant has an immediately available source of CEL that can at least partially compensate for its otherwise low endogenous production ${ }^{(16)}$. The lack of CEL in infant formula partly explains why premature infants may encounter incomplete fat digestion when administered standard infant fat formula ${ }^{(17,18)}$. One of the main components of the resultant incompletely digested fat in the intestine of the premature infant is NEFA, whose persistence in the lumen of the premature gastrointestinal tract may accumulate within cells and disrupt the cell membrane ${ }^{(19)}$, leading to significant toxicity to the enterocyte ${ }^{(20)}$. Supplementation of preterm formula with PUFA has been shown to reduce the incidence of NEC in animal models, which has been linked to the ability of PUFA to suppress TLR4 and platelet-activating factor receptor gene expression; these molecules are important in the pathogenesis of NEC in epithelial cells ${ }^{(4)}$. However, although these studies have focused on the potential role of unsaturated fatty acids in the pathogenesis of NEC, the potential roles - if any - of using hydrolysed or pre-digested fat (PDF) for NEC prevention to essentially bypass the lack of CEL in the gastrointestinal tract of the premature infant remain incompletely understood.

On the basis of these findings, we hypothesise that incomplete fat digestion in the premature intestine leads to intestinal inflammation and the development of NEC, partly owing to the accumulation of undigested TAG-containing unsaturated fatty acids within the epithelium of the distal ileum, leading to the accumulation of reactive oxygen intermediates, which induces an inflammatory response. We sought to test this hypothesis by studying the extent of NEC development using (a) standard infant formula, (b) PDF and (c) a very low fat formula, the nutritional components of which are described in Table 1.

\section{Methods}

\section{Chemical reagents}

Reagents were obtained from the following sources: 'Oil Red O' kit (catalogue no. ab150678; Abcam), RNeasy ${ }^{\circledR}$ kit (catalogue no. 74106; Qiagen) and QuantiTect ${ }^{\circledR}$ Reverse Transcription (catalogue no. 205313; Qiagen), dihydroethidium (DHE, catalogue no. D7008; Sigma), 4',6-diamidino-2-phenylindole, dihydrochloride (DAPI, catalogue no. D9542; Sigma) and CEL (BSDL) ELISA kit (catalogue no. MBS098208; MyBiosource, Inc.) (forward and reverse primers) (custom designed using NCBI Primer-BLAST online program and ordered from Integrated DNA Technologies).

\section{Animal study approval}

All experiments involving mice described in this study were carried out in accordance with the recommendations in the Guide for the Care and Use of Laboratory Animals of the National Institutes of Health, and are approved by Johns Hopkins University protocol, according to The ARRIVE Guidelines of the 'NC3R' ${ }^{(21)}$. C57BL/6J mice were purchased from the Jackson laboratory and bred in the pathogen-free facility at Johns Hopkins University for multiple generations to stabilise intestinal microbiota. All mice were given ad libitum access to water, food and housed in a temperature-controlled room $\left(22^{\circ} \mathrm{C}\right)$ with $12 \mathrm{~h}$ light $-12 \mathrm{~h}$ dark cycles. All mice used in the study were euthanised humanely using isoflurane anaesthesia by inhalation (approximately 3-4\% isoflurane), followed by cervical dislocation.

\section{Induction of necrotising enterocolitis in neonatal mice}

Experimental NEC was induced in 7-8-d-old (approximately $3.0 \mathrm{~g}$ body weight) neonatal mouse pups as previously described $^{(22-24)}$. Neonatal pups were randomly divided into control and treatment groups and experimental models were repeated at least three times with eight or more mice per treatment group. Neonatal pups were gavage fed $(40 \mu \mathrm{l} / \mathrm{g})$ five times per $\mathrm{d}$ (07.00-19.00 hours) with formula containing one of three types of fat (described below and illustrated in Fig. 1), which was supplemented with bacterial stock that had been cultured from the stool of an infant with severe NEC $(12.5 \mu \mathrm{l}$ of stool slurry in $1 \mathrm{ml}$ of formula). The stool mixed formula $(50 \mu \mathrm{l} / \mathrm{g}$ of mouse body weight ${ }^{(25)}$ was administered using a 24 -French angiocatheter placed into the mouse oesophagus. Mice were exposed to hypoxia $\left(5 \% \mathrm{O}_{2}, 95 \% \mathrm{~N}_{2}\right)$ for $10 \mathrm{~min}$ in a chamber (Billups-Rothenberg Inc.), twice daily (07.00 and 13.00 hours immediately after feeding) for $4 \mathrm{~d}$, and the bacterial slurry was added to the formula on each day. Additional breast-fed control groups, which were exposed to hypoxia only, bacteria only and hypoxia + bacteria, and formula-fed groups, which were exposed to formula only, formula + bacteria (no hypoxia) and formula + hypoxia (no bacteria), were also included to evaluate the role of the individual components of the model for the development of intestinal inflammation. For mice in the breastfed/hypoxia alone group, hypoxia $\left(5 \% \mathrm{O}_{2}, 95 \% \mathrm{~N}_{2}\right)$ was given for $10 \mathrm{~min}$, twice daily for $4 \mathrm{~d}$ (at 07.00 and 13.00 hours), and immediately returned with dams similar to NEC-treated groups. For the breast-fed/bacteria control group, mice were gavage-fed 
Table 1. Nutritional composition of study formulations ${ }^{\star}$

\begin{tabular}{|c|c|c|c|}
\hline & $\begin{array}{l}\text { Standard formula } \\
\text { (per litre) }\end{array}$ & $\begin{array}{l}\text { PDF formula } \\
\text { (per litre) }\end{array}$ & $\begin{array}{l}\text { Very low fat formula } \\
\text { (per litre) }\end{array}$ \\
\hline Protein $(\mathrm{g})$ & $56 \cdot 5$ & $57 \cdot 1$ & $58 \cdot 1$ \\
\hline Fat $(\mathrm{g})$ & 71 & 71 & 3.7 \\
\hline High-oleic safflower oil (g) & 27.66 & 24.75 & 0 \\
\hline Soya oil (g) & $20 \cdot 7$ & 0 & 0 \\
\hline Coconut oil (g) & $19 \cdot 8$ & 10.5 & 0 \\
\hline Monoacylglycerol palmitate $(\mathrm{g})$ & 0 & 14.25 & 0 \\
\hline Soyabean oil NEFA (g) & 0 & $12 \cdot 4$ & 0 \\
\hline Lecithin $(\mathrm{g})$ & 0 & $7 \cdot 3$ & 0 \\
\hline Distilled monoacylglycerols (g) & 1.5 & 0 & 0 \\
\hline $\mathrm{DHA}(\mathrm{mg})$ & 146 & 135 & 161 \\
\hline ARA (mg) & 312 & 322 & 355 \\
\hline Carotenoids (mg) & 59 & 59 & 61 \\
\hline $\begin{array}{l}\text { Remaining fat from proteins, carrier oils } \\
\text { from vitamins, carotenoids, DHA and ARA (g) }\end{array}$ & 0.82 & 1.28 & $3 \cdot 12$ \\
\hline Carbohydrate (g) & 62 & 63 & 138 \\
\hline \multicolumn{4}{|l|}{ Vitamins } \\
\hline A (palm; mg) & 3.69 & 4.00 & 3.35 \\
\hline $\mathrm{E}(\mathrm{mg})$ & 18 & 18 & 19 \\
\hline$C(\mathrm{mg})$ & 375 & 375 & 375 \\
\hline $\mathrm{B}_{1}(\mathrm{mg})$ & $2 \cdot 45$ & $2 \cdot 25$ & 2.45 \\
\hline $\mathrm{B}_{2}(\mathrm{mg})$ & $6 \cdot 1$ & 6.02 & $6 \cdot 7$ \\
\hline $\mathrm{B}_{6}(\mu \mathrm{g})$ & 783 & 802 & 832 \\
\hline $\mathrm{B}_{12}(\mu \mathrm{g})$ & $10 \cdot 2$ & $10 \cdot 1$ & 11.4 \\
\hline Pantothenic acid (mg) & 12 & $12 \cdot 38$ & $12 \cdot 7$ \\
\hline Folic acid $(\mu \mathrm{g})$ & 334 & 297.4 & 328.2 \\
\hline Niacin $(\mathrm{mg})$ & $16 \cdot 4$ & $16 \cdot 22$ & 17 \\
\hline Biotin $(\mu \mathrm{g})$ & 118 & $115 \cdot 4$ & 125.9 \\
\hline Total choline (mg) & 317 & 474 & 341 \\
\hline \multicolumn{4}{|l|}{ Minerals } \\
\hline $\mathrm{Na}(\mathrm{mg})$ & 762 & 755 & 802 \\
\hline $\mathrm{K}(\mathrm{mg})$ & 2228 & 2319 & 2539 \\
\hline Chloride (mg) & 1175 & 1175 & 1184 \\
\hline $\mathrm{Ca}(\mathrm{mg})$ & 2402 & 2371 & 2432 \\
\hline$P(\mathrm{mg})$ & 1269 & 1362 & 1280 \\
\hline$M g(\mathrm{mg})$ & 154 & 158.1 & $158 \cdot 2$ \\
\hline $\mathrm{Fe}(\mathrm{mg})$ & 20 & 20.2 & 21.2 \\
\hline Zn (mg) & $13 \cdot 1$ & 12.69 & $13 \cdot 77$ \\
\hline $\mathrm{Cu}(\mathrm{mg})$ & $1 \cdot 13$ & 1.09 & $1 \cdot 18$ \\
\hline
\end{tabular}

bacteria (equal amount of bacteria given to NEC-treated mice that is $10 \mu \mathrm{l}$ of NEC bacterial stock slurry, diluted in $100 \mu \mathrm{l}$ of saline/pup) once daily for $4 \mathrm{~d}$. We and others have previously demonstrated that under administration of the standard formula, this experimental model induces significant intestinal inflammation expression of pro-inflammatory cytokines, IL-6, IL-1 $\beta$ and TNF- $\alpha$ that closely mimics human NEC ${ }^{(26,27)}$. To determine the effect of the antioxidant on reactive oxygen species (ROS) generation, NEC formula was supplemented with $\mathrm{N}$-acetylcysteine (NAC) $(100 \mathrm{mg} / \mathrm{kg})$ and administered to mice in the NEC model. Control mice were gavage-fed once daily with a similar dose of $100 \mathrm{mg} / \mathrm{kg}$ of NAC.

\section{Nutritive formulas}

Three different study formulas were used, each with varying types and amounts of fats (manufactured by Abbott Laboratories) and which contained identical ingredients except for the fat composition. Standard formula was composed of $100 \%$ TAG-rich oils (mixture of 39\% high oleic safflower oil (HOSO), $29 \%$ soya oil and $27.9 \%$ coconut oil), and these are described in detail in Table 1. PDF formula contained approximately $50 \%$ TAG composed of a mixture of soyabean NEFA (17.5\%), 2-monoacylglycerol palmitate (20\%) and phospholipid lecithin (10.3\%) along with HOSO (34.8\%) and coconut oil (14.8\%). Soyabean NEFA were added into the formula in the Ca salt form where the NEFA were first mixed with calcium hydroxide during manufacturing. Very low fat formula was formulated with no added TAG except DHA and ARA and lipid-soluble vitamins to match the standard and PDF formulas. In an attempt to close the gap in energetic content of the low-fat formula as compared with the other formulas, lactose was used to replace the fat energy, and energetic values were similar between the three formulas. All three study formulas were manufactured using the same homogenisation and thermal treatment conditions, which are representative of commercial ready to feed formulas. The nutrition profiles of the formulas are shown in Table 1 and the experimental scheme for the NEC model is shown in Fig. 1. The breast-fed (control) animals remained with their mothers and received breast milk ad libitum. 
(a)

Experimental scheme

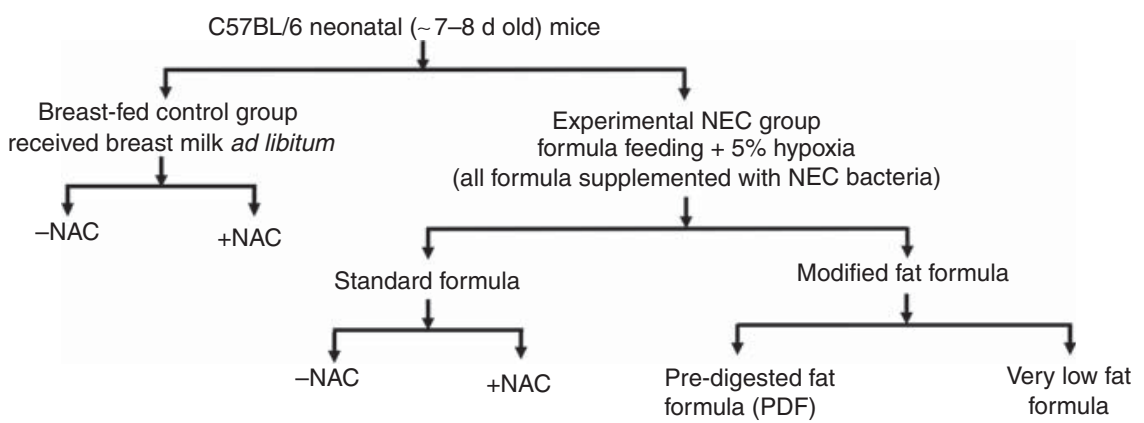

(b)

Illustration of location of tissue sampling

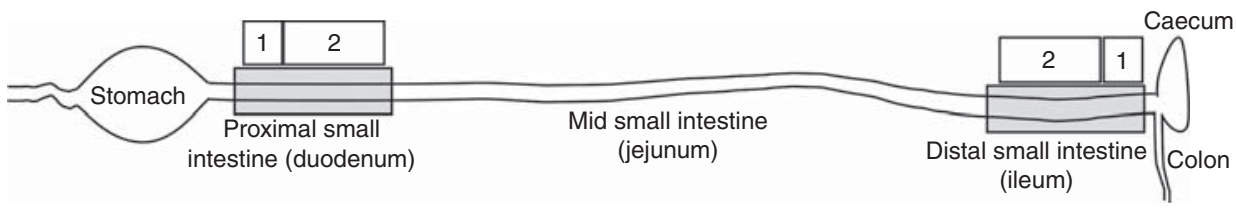

$1=$ tissue for RNA isolation

$2=$ tissue for histology

Fig. 1. Experimental scheme (a) and location of tissue sampling (b) used in the current studies. NAC, $N$-acetylcysteine; NEC, necrotising enterocolitis.

\section{Necrotising enterocolitis severity assessment}

NEC severity was determined based upon a validated scoring system applied to de-identified, paraformaldehyde (PFA)-fixed/ paraffin-embedded/haematoxylin and eosin (H\&E)-stained intestinal sections from both proximal and distal intestine in consultation with a paediatric pathologist who was blinded to the group allocation. Histological NEC severity score was assigned as described previously ${ }^{(28,29)}$ : 0 (no injury), 1 (minor-submucosal, lamina propria separation), 2 (moderate separation of submucosa ${ }^{(28)}$, lamina propria and oedema in submucosal and muscular layers) and 3 (severe separation of submucosa, lamina propria, severe oedema and villous sloughing or loss of villi).

\section{Ontogeny of transcript levels of carboxyl ester lipase in pancreas by quantitative real-time $P C R$}

To measure the transcripts levels of CEL, also called bile-saltdependent lipase (BSDL), within the pancreas of breast-fed neonatal (postnatal day, p2) to weanling (postnatal day, p15) mice by quantitative real-time PCR (qRT-PCR), whole pancreas was carefully dissected out under a dissection stereoscope (Nikon; Nikon Instruments Inc.) and snap-frozen until processing for total RNA isolation and qRT-PCR assay as described below.

\section{Ontogeny of carboxyl ester lipase protein levels by ELISA assay}

To assess the ontogeny of CEL by ELISA, the stomach content of neonatal mice (postnatal days 2, 5, 10 and 15) was collected immediately after euthanasia and snap-frozen in liquid $\mathrm{N}_{2}$. CEL in stomach contents was measured using mouse lipase CEL (i.e. BSDL) ELISA kit, as per the manufacturer's instructions.

\section{Quantitative real-time PCR}

Total RNA was isolated from snap-frozen whole intestine (approximately $1 \mathrm{~cm}$ length) and pancreas using RNeasy ${ }^{\circledR}$ kit, and checked for RNA purity and concentration on SpectraMax ${ }^{\circledR}$ microplate reader (Molecular Devices). A measure of $0.5 \mu \mathrm{g}$ of total RNA was reverse-transcribed for complementary DNA synthesis using the QuantiTect ${ }^{\circledR}$ Reverse Transcription kit. qRTPCR was then performed on a Bio-Rad CFX96 Real-Time System (Bio-Rad Labs) using Sybr green mix (Bio-Rad Labs), forward and reverse primers (Table 2 ). The mRNA expression relative to the housekeeping gene ribosomal protein large P0 (RplpO) was calculated using the $2^{-\Delta \Delta C_{t}}$ method as described ${ }^{(30)}$.

\section{'Oil Red O' staining for lipid droplets}

To assess the presence of intracellular lipid uptake, we performed 'Oil Red O' staining on proximal and distal intestinal tissues harvested $45 \mathrm{~min}$ after formula or breast milk feeding. To do so, fresh intestinal tissues were fixed with $4 \%$ PFA for $4 \mathrm{~h}$, immersed in $30 \%$ sucrose solution for $2 \mathrm{~d}$ and frozen blocks were prepared in TissueTek media and cryomolds. Cryo-sections measuring $10 \mu \mathrm{m}$ were then cut using a Cryostat (Thermo Fisher), dehydrated in PBS and stained for lipid droplets using the 'Oil Red O' kit according to the manufacturer's instructions. Sections were briefly counter-stained with haematoxylin (Modified Mayer's), mounted using Gelvetol mount media and imaged using the EVOS imaging system (Invitrogen). Cryo-tissue sections were also prepared without PFA fixation for 'Oil Red O' staining, and no difference in staining was observed, but tissue integrity was far superior in PFA-fixed tissues; therefore, all data were collected from PFA-fixed cryo-sections. 
Table 2. Primer sequences

\begin{tabular}{|c|c|c|c|}
\hline Genes & Forward sequence & Reverse sequence & Amplicon size (bp) \\
\hline Akr1b7 & GGTGGTGATCCCCAAGTCTG & GCССTCCAGTTCCTGTTGAA & 120 \\
\hline$C E L(B S D L)$ & ACGATAACCAGCGCTTCCAT & TCATCCTCAGGGGGAGTGAG & 125 \\
\hline Gpx2 & TCAATGGGCAGAACGAGCAT & CGCACGGGACTCCATATGAT & 118 \\
\hline$I L-1 \beta$ & AGTGTGGATCCCAAGCAATACCCA & TGTCCTGACCACTGTTGTTTCCCA & 175 \\
\hline Keap1 & CTCAACCGCTTGCTGTATGC & TTCAACTGGTCCTGCCCATC & 194 \\
\hline MPO & GACAGTGTCAGAGATGAAGCTACT & TTGATGCTTTCTCTCCGCTCC & 189 \\
\hline Nox2 & GACACGCATGCCTTTGAGTG & TGCACAGCAAAGTGATTGGC & 143 \\
\hline Nqo1 & GGTAGCGGCTCCATGTACTC & CCAGACGGTTTCCAGACGTT & 198 \\
\hline Nrf2 & AGCACTCCGTGGAGTCTTCCATTT & TGTGCTTTAGGGCCGTTCTGTTTG & 115 \\
\hline$T N F-a$ & TTCCGAATTCACTGGAGCCTCGAA & TGCACCTCAGGGAAGAATCTGGAA & 144 \\
\hline Rplpo & GGCGACCTGGAAGTCCAACT & CCATCAGCACCACAGCCTTC & 143 \\
\hline
\end{tabular}

Akr1b7, aldo-keto reductase family 1, member B7; $C E L$, carboxyl ester lipase; $B S D L$, bile-salt-dependent lipase; Gpx2, glutathione peroxidase 2; Keap1, kelch-like ECH-associated protein 1; MPO, myeloperoxidase; Nox2, NADPH oxidase; Nqo1, NAD(P)H quinone dehydrogenase 1; Nrf2, nuclear factor erythroid 2-related factor; RplpO, ribosomal protein large PO.

\section{Immunohistochemistry and haematoxylin-eosin staining}

Specific parts of proximal and distal intestinal tissues as illustrated in Fig. 1 were obtained for histological examination. For the detection of ROS accumulation in mouse intestinal tissue, we used the DHE oxidation staining method, which was based on its oxidation to ethidium in the presence of ROS, which then intercalates into DNA producing bright red nuclear fluorescence (Sigma). In brief, $10-\mu \mathrm{m}$ cryo-sections were hydrated in PBS and stained with $5 \mu \mathrm{m}$ DHE for $30 \mathrm{~min}$ in the dark, the nuclei were counter-stained with DAPI, mounted using Gelvetol media and immediately imaged using Nikon A1 confocal microscope (Nikon). H\&E staining was performed on $4 \%$ PFA-fixed/paraffin-embedded sections (5- $\mu \mathrm{m}$ thickness). Malondialdehyde (MDA) immunofluorescence staining was performed as described ${ }^{(31)}$.

\section{Statistical analysis}

Statistical analysis was performed using PRISM version 7.0 (Graph Pad). The pups were randomised to the treatment group, and blinded analyses were performed using either Fisher's exact test or ANOVA with multiple comparisons, and post hoc analyses were performed whenever statistical differences were determined in the multiple group analyses. Statistical significance was accepted at $P<0.05$. On the basis of the predicted effects of both the PDF system and the very low-fat formula, with an $\alpha$ error of 0.05 and a $\beta$ error of $0 \cdot 10$, in order to assess a $50 \%$ reduction in cytokine expression by RT-PCR, ROS generation and ELISA expression of CEL, we calculate a sample size of eight pups in each group, which is also the expected survival rate based upon technical variability, between litters.

\section{Results}

\section{Establishment of a model of necrotising enterocolitis in} newborn mice and determination of appropriate controls for assessment of infant formula

We first sought to develop a platform for the assessment of different fat compositions in experimental NEC in newborn mice, and thus evaluated the relative contribution of each of the individual components - namely the administration of infant formula, the induction of hypoxia and the administration of the cultured bacterial slurry from an infant with severe NEC - to the development of intestinal inflammation. As shown in Fig. 2, newborn mice that were exposed to breast milk and either hypoxia or bacterial slurry did not develop NEC, as revealed by gross examination of the intestine, intestinal histology and qRTPCR analysis of pro-inflammatory gene expression in the intestinal mucosa (Fig. 2(a) and (c)). Similarly, mice that were administered 'standard fat'-containing formula feeds along with either hypoxia or bacteria had no evidence of NEC (Fig. 2(b) and (c)). By contrast, mice that were administered the combination of 'standard fat'-containing formula feeds, hypoxia and bacteria developed severe NEC as revealed by the presence of air within the wall of the bowel (Fig. 2(b) iv), histologic evidence of mucosal disruption (Fig. 2(b) viii) and the induction of pro-inflammatory genes in the distal ileum (Fig. 2(c)). These findings reveal that the induction of NEC requires a combination of formula feeding, bacterial supplementation and exposure to hypoxia together. Subsequent studies were thus designed in which NEC was induced using these three variables, and in which only the fat component of the formula was varied, in comparison with control mice that were left with their mothers and were breast-fed. This approach allowed us to determine the effect of fat type on the development of NEC, as examined below.

The administration of formula containing 'pre-digested' fat attenuates the severity of necrotising enterocolitis in mice

We next administered formulas containing varying fat compositions to newborn mice and assessed the effects on NEC severity. All formulas contained approximately $71 \mathrm{~g}$ of the various types of fat, except the 'very low fat' formula, which contained $3.7 \mathrm{~g}$ of fat; formulas contained approximately $56.5-$ $58.1 \mathrm{~g}$ of protein per litre (Table 1), which approximates that seen in rodent milk ${ }^{(32)}$. The 'standard' formula contained $100 \%$ TAG in the form of high oleic safflower, soyabean and coconut oils, as shown in Table 1. As shown in Fig. 3, and using an evaluation scheme that was consistent with our prior stu$\operatorname{dies}^{(5,26,33)}$, the administration of this 'standard fat formula' to newborn mouse pups resulted in progressive weight loss (red curve in Fig. 3(A)) in comparison with breast-fed mice (blue curve in Fig. 3(A)), and the development of severe mucosal 
(a)

Breast fed (-) hypoxia, (-) bacteria

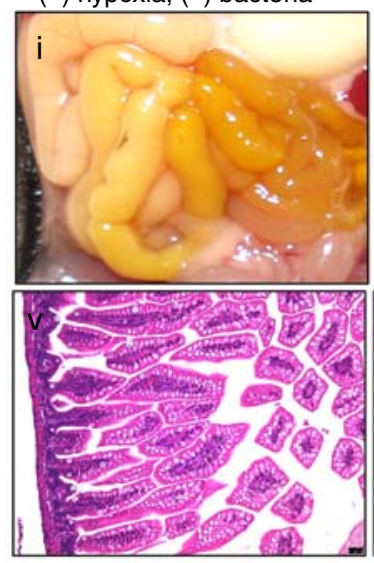

(b)
Breast fed

(+) hypoxia (-) bacteria

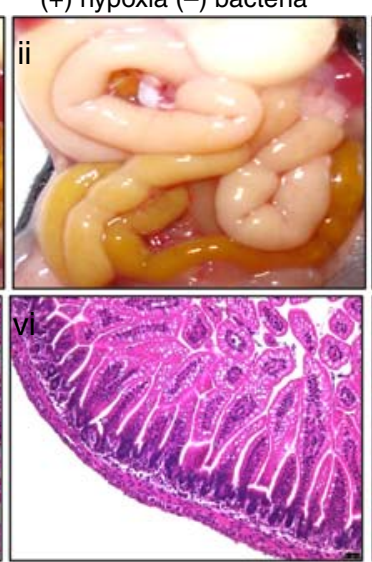

Breast fed

$(-)$ hypoxia (+) bacteria

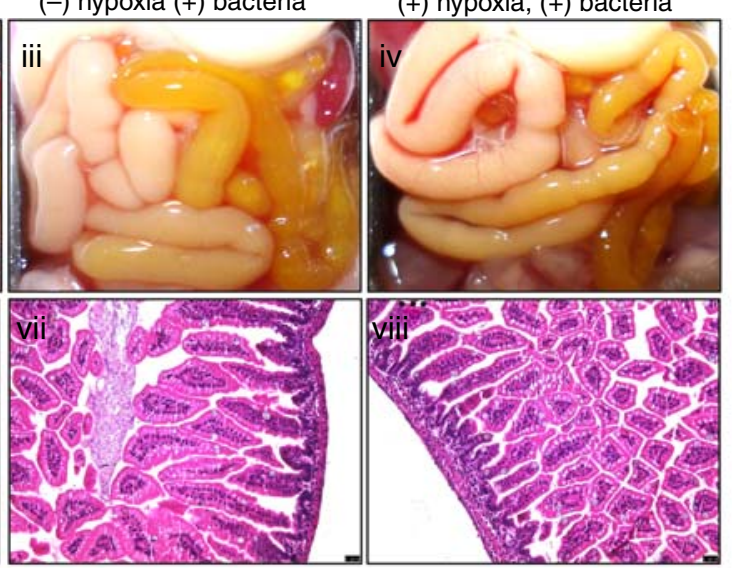

Formula fed

(-) hypoxia, (-) bacteria

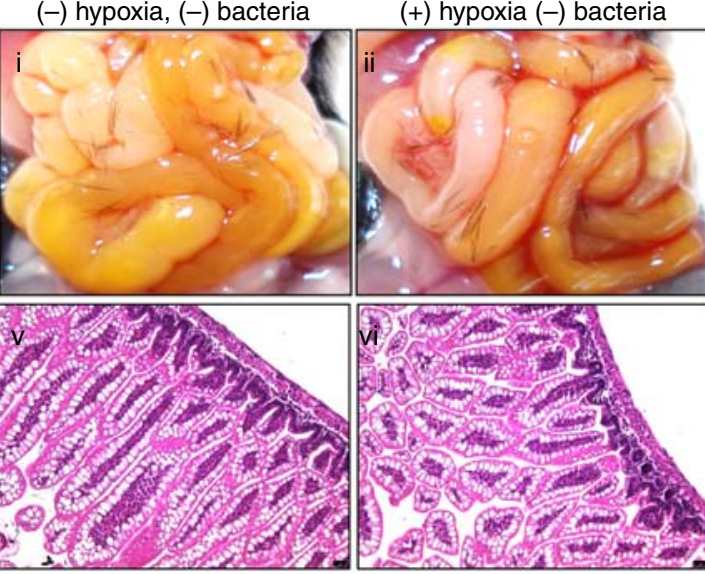

Formula-fed mice +/- hypoxia +/- bacteria

Formula fed

Formula fed $(-)$ hypoxia $(+)$ bacteria



Formula fed (+) hypoxia, (+) bacteria

(c) Proinflammatory cytokines - breast-fed and formula-fed groups
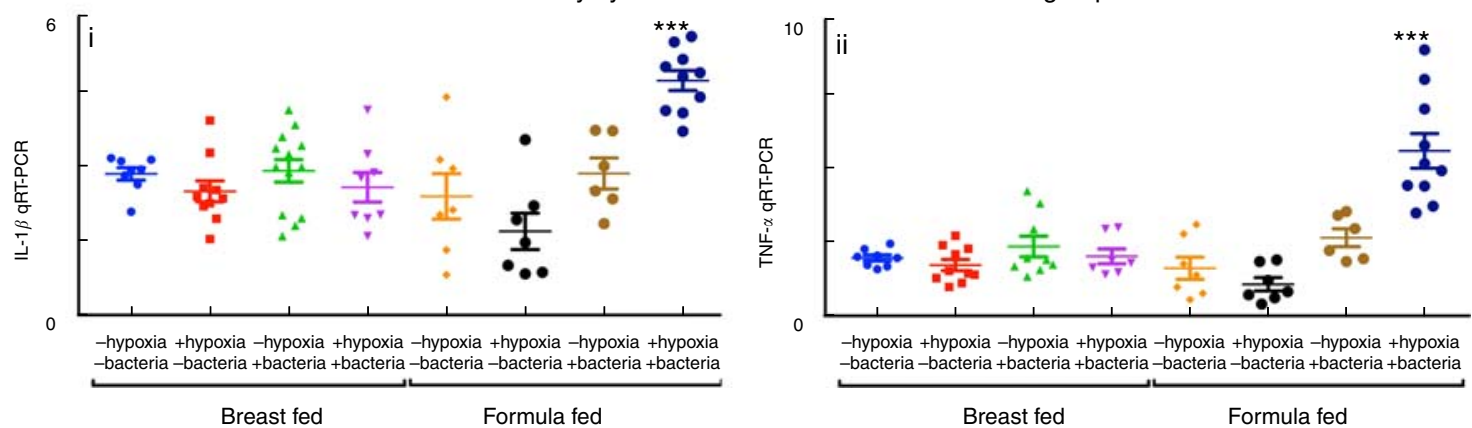

Fig. 2. Establishment of a model of necrotising enterocolitis in newborn mice and determination of appropriate controls for assessment of infant formula. (a,b) Results from representative distal ileal samples of mice subjected to breast-feeding and formula feeding are shown. (a) Gross morphology (a; i-iv), haematoxylin-eosin (H\&E)stained histology (a; v-viii) of breast-fed groups. (b) Gross morphology (b; i-iv), H\&E-stained histology (b; v-viii) of formula-fed groups. (c) Levels of pro-inflammatory cytokines, IL-1 $\beta$ (c; i) and TNF- $\alpha$ (c; ii). qRT-PCR, quantitative real-time PCR. ${ }^{* \star *} P<0.0001$.

injury and marked morphological change to the small intestine, characterised by oedema, air within the bowel wall (pneumatosis intestinalis) and patchy intestinal necrosis (Fig. 3(C) and (G)) consistent with the findings in Fig. 2. These morphologic changes were accompanied by an increase in pro-inflammatory cytokines IL- $1 \beta$ and TNF- $\alpha$ (Fig. 3(I)), and an increase in the NEC severity score (Fig. 3(K)). Breast-milk-fed control mice showed normal gross morphology (Fig. 3(B)), intact architecture of the intestinal epithelium (Fig. 3(F)) and low expression of pro-inflammatory cytokines (Fig. 3(G)). On the basis 
(A)
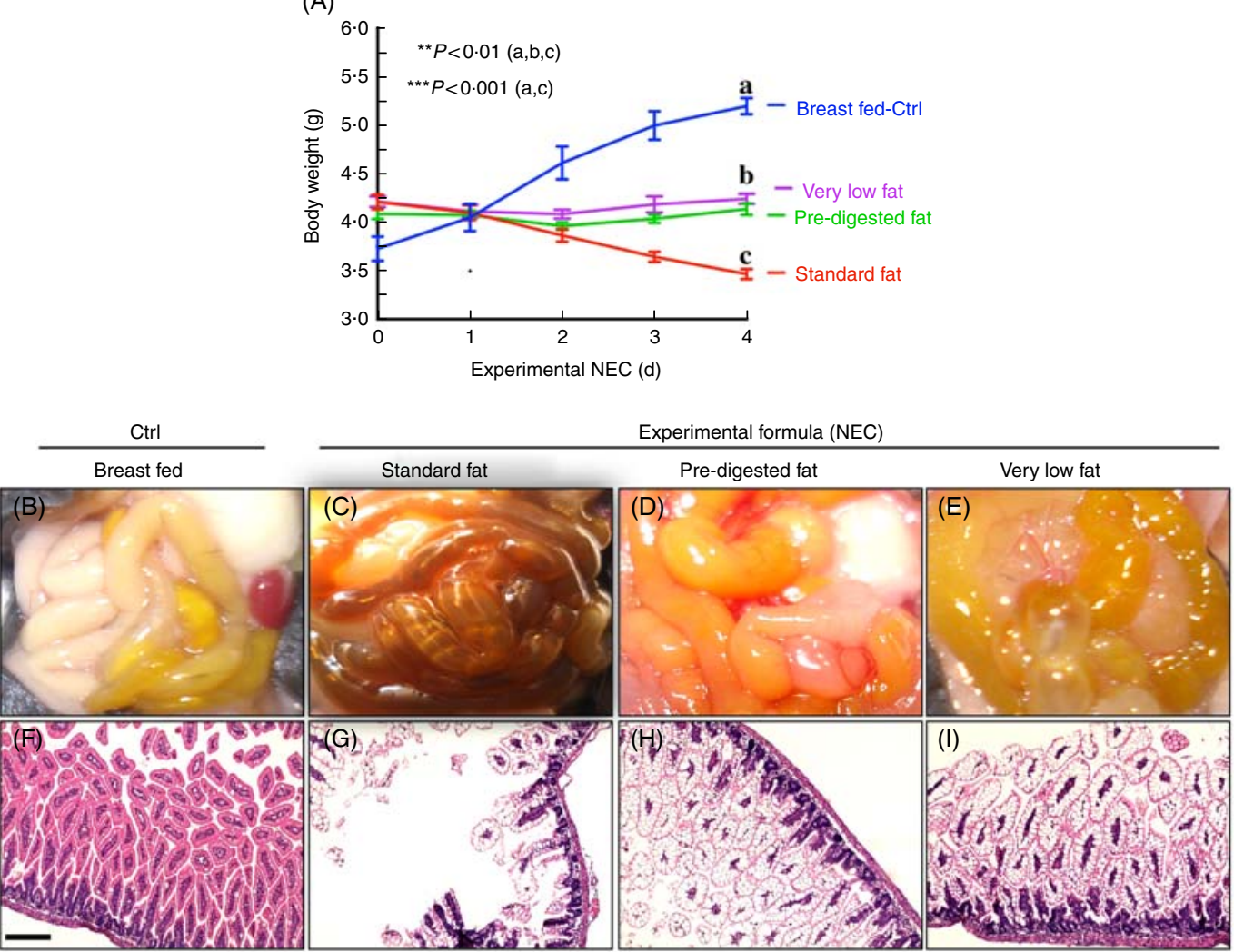

(J)

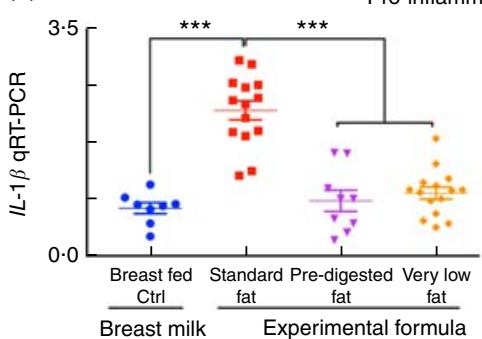

ory cytokines

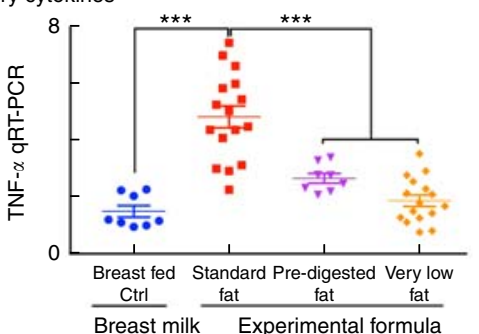

(K)

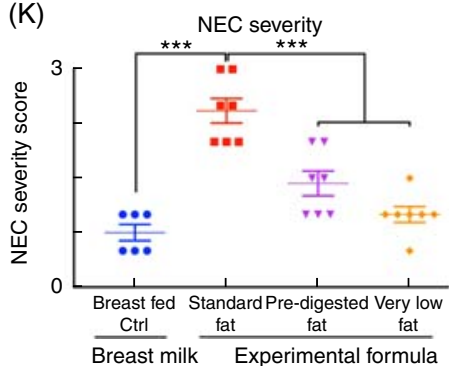

Fig. 3. Administration of 'pre-digested fat'-containing formula to neonatal mice reduces the incidence and severity of experimental necrotising enterocolitis (NEC). (A) Body weights. (B-E) Photomicrographs of gross images. (F-I) Haematoxylin-eosin (H\&E)-stained images. (J) Quantitative real-time PCR (qRT-PCR) of pro-inflammatory cytokines. (K) NEC severity scores, in neonatal mice not subjected to treatment (Ctrl, control-breast-fed) or experimental NEC treatments. ${ }^{* \star} P<0 \cdot 01$ $(\mathrm{a}, \mathrm{b}, \mathrm{c}),{ }^{\star \star \star} P<0.001(\mathrm{a}, \mathrm{c})$, each dot in dot-graphs represents data from an individual mouse, scale bar $=10 \mu \mathrm{m}$.

of these findings, we next hypothesised that the removal of long-chain TAG from the formula would reduce NEC severity. To test this specifically, we administered a formula that was deficient in long-chain TAG oil, which we termed 'very low fat' formula, which resulted in less weight loss (pink curve in Fig. 3(A)), less mucosal and gross morphological injury (Fig. 3 (E) and (I)), reduced pro-inflammatory cytokine expression (Fig. 3(J)) and reduced NEC severity scores (Fig. 3(K)), as compared with mice in the 'standard fat' formula group. To further assess the role of fat in NEC development, we next administered formula with reduced TAG-containing fat in a 'PDF' composition, which was not dependent upon the action of lipases within the lumen of the intestine for digestion. Specifically, 'PDF'-containing formula (i.e. 'PDF formula') contains approximately $50 \%$ long-chain TAG and a mixture of soyabean oil NEFA, monoacylglycerol palmitate and soya lecithin compared with $100 \%$ long-chain TAG fats that are present in the 'standard fat' formula. As shown in Fig. 3, the administration of 'PDF' (which contains DHA and ARA at concentrations similar to those of standard formula) resulted in marked preservation of infant weight (green curve in Fig. 3(A)), and significantly reduced gross morphological injury (Fig. 3 (D)), histological injury (Fig. 3(H)), pro-inflammatory cytokine expression (Fig. 3(J)) and NEC severity (Fig. 3(K)). Importantly, there was no difference observed in body weight loss, gross morphology of the intestine, histological examination of the terminal ileum, expression of pro-inflammatory cytokines and NEC severity scores between the 'PDF' and 'very low fat' formula groups. Taken together, the above findings suggest that the type of fat used in formula may 
contribute to NEC pathogenesis, leading us to next determine the potential mechanisms involved.

\section{Pancreatic carboxylic ester lipase is reduced at the time of necrotising enterocolitis development in mice}

The content of lipases in the lumen of the intestine can be assessed by measurement of both pancreatic and gastric lipases. To determine whether lipases are reduced in the intestine of newborn mice at the time of NEC development, we assessed the expression of carboxyl ester lipase within the pancreas of neonatal to weaned breast-fed mice by qRT-PCR, and also measured the amount of CEL (also called BSD-lipase) in the gastric contents of mice at varying postnatal ages from days 2 through 15 by ELISA ${ }^{(15)}$. As shown in Fig. 4(a), the pancreatic expression of CEL was naturally low immediately after birth and increased significantly over time. These findings were supported by the observation shown in Fig. 4(b) that the concentration of CEL is also significantly greater in the gastric contents of mice as they mature towards postnatal day 15 as compared with early newborn mice. Not surprisingly, the composition of fat within the administered formula had no effect on the expression of individual lipases (measured at the end of the experimental NEC model), as mice that were fed 'standard fat' formulas were found to have similar expression of CEL as compared with mice that were fed either 'PDF' or 'very low fat' formula (Fig. 4(c)). We therefore next sought to investigate the potential effects of impaired lipase content of the newborn mouse intestine on the accumulation of fat within the proximal and distal intestinal epithelium

\section{Administration of formula containing 'pre-digested fat'} results in reduced accumulation of lipid and reactive oxygen species generation in the intestinal mucosa as compared with standard infant formula

Having shown reduced expression of lipases within the pancreas and stomach of the developing mice, and given the potential cytotoxic effects that non-digested fats may exert on the ileal mucosa ${ }^{(15)}$, we next sought to evaluate the effects - if any - on the accumulation of fat within the intestinal epithelium of mice subjected to models of NEC using formulas containing various amounts of fat as in Table 1. As shown in Fig. 4, there was a significant accumulation of lipid droplets in the duodenal epithelium of mice that received breast milk (Fig. 4(d)), as well as the three infant formulas tested, as determined by 'Oil Red O' staining (Fig. 4(e-g)). By contrast, examination of the distal ileum - where NEC-induced injury most commonly develops revealed that the administration of 'standard fat'-containing formula (Fig. 4(i)) to newborn mice resulted in significantly increased lipid accumulation in the intestinal epithelium, as compared with breast-fed control mice (Fig. 4(h)) and with mice that were administered a 'PDF' formula (Fig. 4(j)) or a 'very low fat' formula (Fig. 4(k)). To assess the potential link between intracellular fat accumulation and ROS-induced mucosal injury, we next sought to measure ROS accumulation in both the proximal and distal intestine by staining the intestinal tissue with the ROS-sensitive fluorescent dye $\mathrm{DHE}^{(27)}$. Examination of the duodenum of mice that were either breast-fed or that were fed 'standard fat'- or 'PDF'-containing formula revealed the accumulation of intracellular 'Oil Red O'-stained fat droplets (Fig. 4(d-g)) - yet very little nuclear DHE staining (Fig. 5(a) and (d)). By contrast, the administration of 'standard fat'-containing formula resulted in the marked accumulation of ROS as manifested by increased DHE staining (Fig. 5(f)) corresponding to the accumulation of intracellular 'Oil Red O'-stained fat droplets (Fig. 4(i)) in the distal intestine (ileum), suggesting a link between lipid accumulation and ROS generation in the distal intestine, where NEC disease normally develops. Interestingly, in the distal intestine (ileum), the administration of either a 'PDF'- or a 'very low fat'-containing formula resulted in significantly less accumulation of intracellular 'Oil Red O'-stained fat droplets (Fig. 4(j) and (k)) and ROS accumulation (Fig. 5(g) and (h)) as compared with the 'standard formula' group (Fig. 4(i) and 5(f)). On the basis of these findings, we next sought to evaluate the degree of lipid peroxidation of polyunsaturated lipids by ROS, and then sought to determine whether an ROS inhibitor could reduce the incidence of experimental NEC in mice.

The administration of formula containing 'pre-digested fat' leads to a reduction in the accumulation of reactive oxygen species-degraded polyunsaturated lipids in the distal small intestine of mice with necrotising enterocolitis

Given that ROS accumulation can lead to the degradation of polyunsaturated lipids to form reactive electrophile species including $\mathrm{MDA}^{(34)}$, which can cause tissue injury ${ }^{(35)}$, we next measured the extent of MDA accumulation within the intestinal epithelium of newborn mice after exposure to either breast milk or infant formulas containing different types of fat, as described in the 'Methods' section and Table 1. As shown in Fig. 6(a) and (e), breast-fed mice showed low levels of MDA in the duodenum and ileum, consistent with the fact that this diet induces minimal ROS accumulation inside the cells, as described above in Fig. 5(a) and (e). By contrast, examination of the distal small intestine (ileum) of mice that were administered 'standard fat' formula revealed marked accumulation of MDA in the ileum (Fig. 6(f)), consistent with the accumulation of ROS that was detected by DHE staining (Fig. 5(f)), and the histological development of NEC (Fig. 3(c), (g) and (j-k)). Strikingly, the administration of formula containing 'PDF' showed a markedly reduced degree of MDA accumulation in the distal bowel (Fig. 6(g)), similar to that observed in either breast-fed control (Fig. 6(e)) or 'very low fat' formula-fed mice (Fig. 6(h)). Taken together, these findings illustrate that the degree of ROS generation and lipid peroxidation can be influenced partly by the composition of fat (i.e. intact TAG $v$. hydrolysed TAG) provided to the newborn gut.

The administration of $\mathrm{N}$-acetylcysteine with standard infant formula prevents the accumulation of reactive oxygen species and the oxidation of lipids, and reduces the severity of necrotising enterocolitis in newborn mice

Having shown that the fat composition of various infant formulas can influence the degree of accumulation of ROS generation and severity of NEC, we next sought to investigate 

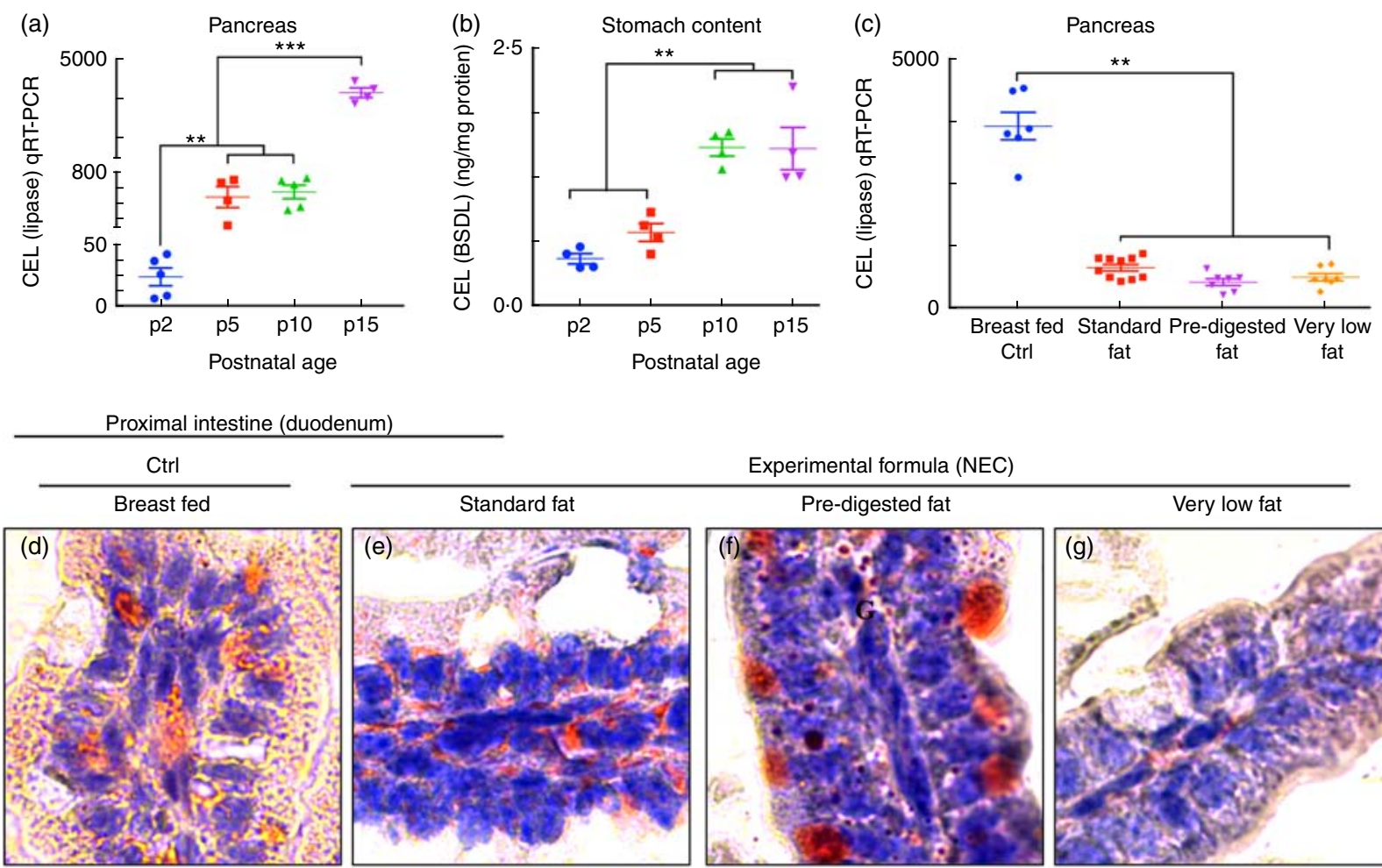

Experimental formula (NEC)
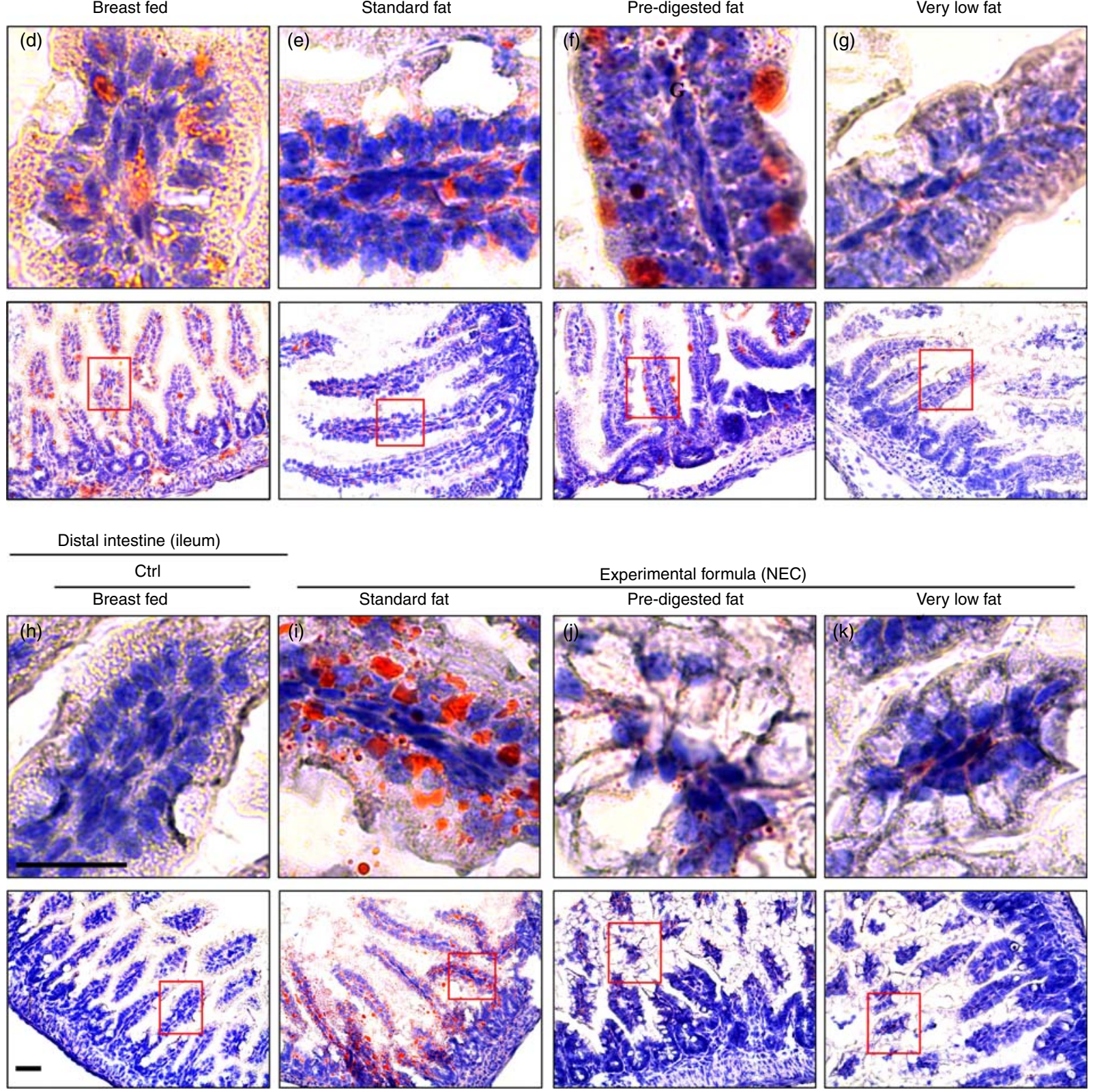

Fig. 4. Low lipase activity in neonatal mice impairs normal fat absorption in the proximal intestine and triggers the accumulation of fat droplets in ileal enterocytes. (a) Ontogeny of carboxyl ester lipase (CEL) (also called bile-salt-dependent lipase (BSDL)) in the pancreas by quantitative real-time PCR (qRT-PCR). (b) CEL/BSDL ELISA. (c) qRT-PCR of CEL in the pancreas of control and necrotising enterocolitis (NEC) mice. (d-k) 'Oil Red O' staining (red droplets) showing counter-stained blue nuclei with haematoxylin stain ( $\mathrm{d}-\mathrm{g}$ proximal intestine and $\mathrm{h}-\mathrm{k}$ distal intestine, $10 \mu \mathrm{m}$ cryo-sections) (Ctrl, control-breast-fed or experimental NEC treatments with hypoxia and formula feeding). ${ }^{\star \star} P<0.01,{ }^{\star \star \star} P<0.001$, each dot represents data from an individual mouse, scale bar $=10 \mu \mathrm{m}$. 
Proximal intestine (duodenum)
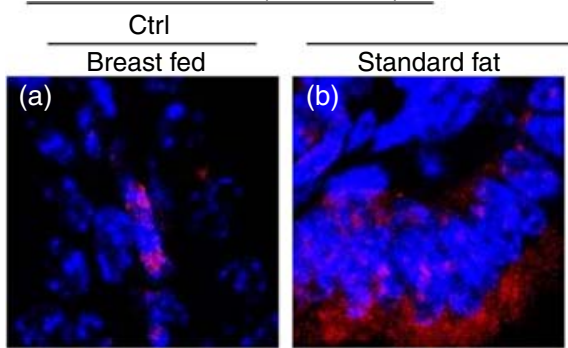

Experimental formula (NEC)
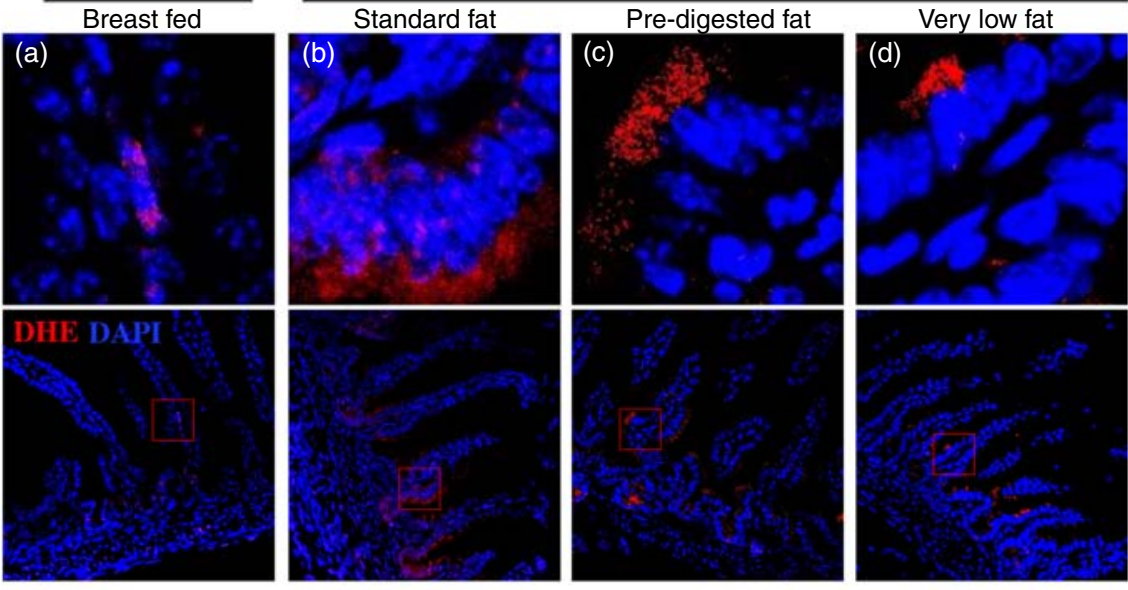

Distal intestine (ileum)


Experimental formula (NEC)
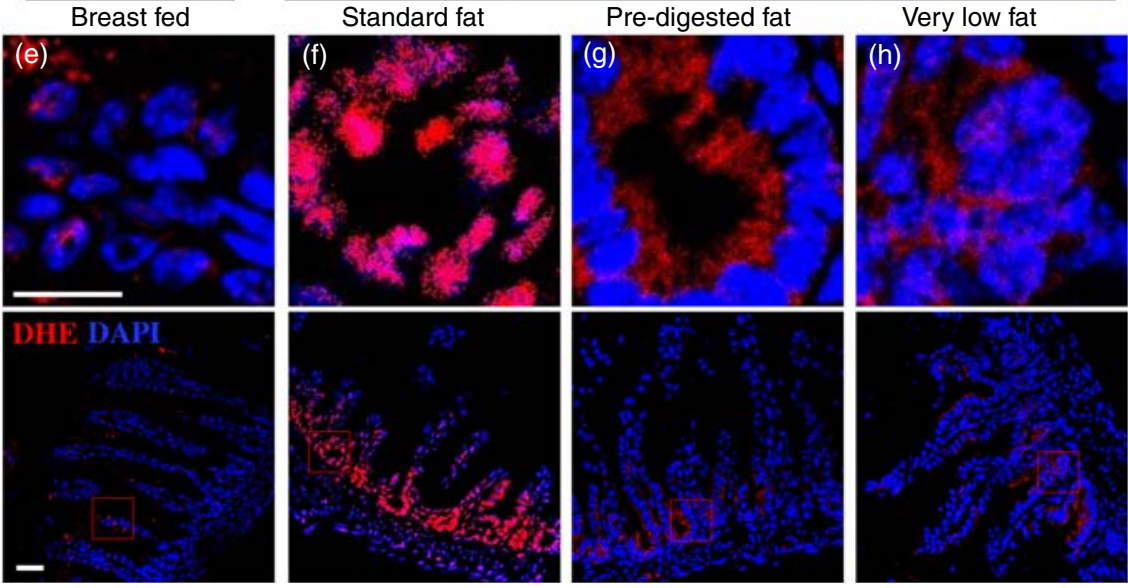

Fig. 5. The effect of fat composition on the accumulation of reactive oxygen species in ileal enterocytes of mice induced to develop necrotising enterocolitis (NEC). $(a-h)$ Immunofluorescence images of dihydroethidium (DHE) (red fluorescence) and 4',6-diamidino-2-phenylindole, dihydrochloride (DAPI) (nuclei, blue) staining from control and NEC mice. (a-d) Proximal (duodenum) and (e-h) distal (ileum), $10 \mu \mathrm{m}$ : cryo-sections (Ctrl, control-breast-fed or experimental NEC treatments with hypoxia and formula feeding). Scale bar $=10 \mu \mathrm{m}$.

whether the use of an ROS quencher could reverse these effects. To do so, we administered NAC to mice that were then induced to develop NEC in the presence of formulas containing various fat compositions. As shown in Fig. 7, the combined oral administration of NAC to the standard fat-containing NEC formula significantly reduced NEC severity as demonstrated by reduced proinflammatory cytokine expression (Fig. 7(a)), as well as preservation of histology of the terminal ileum and NEC severity scores (Fig. 7(b) and (c)). The levels of ROS generation as determined by DHE staining were significantly reduced in the terminal ileum of mice exposed to NEC using NEC formula that was supplemented with NAC (Fig. $7(\mathrm{~d}-\mathrm{g})$ ). Furthermore, the addition of NAC led to a marked reduction in ROS-mediated lipid peroxidation in the terminal ileum, as revealed by reduced MDA staining (Fig. 7(h-k)). We therefore sought to evaluate the mechanisms by which lipid oxidation occurred in the distal intestine, and to determine whether the expression of components of the anti-ROS machinery could play a role in NEC development.
The expression of the anti-reactive oxygen species machinery in the newborn intestine contributes to the pattern of necrotising enterocolitis development in the distal bowel after fat administration

In the final series of studies, we sought to explore the potential mechanisms by which the proximal intestine of mice is protected from NEC-induced injury despite the accumulation of fat in duodenal enterocytes (Fig. 4(e-g)). As shown in Fig. 8(a), the expression levels of the pro-inflammatory cytokines IL- $1 \beta$ and TNF- $\alpha$ were significantly elevated in the ileum but not in the duodenum, consistent with the development of NEC in the more distal regions of the bowel. We thus next studied the expression of genes within the nuclear factor erythroid 2-related factor (Nrf2)/ROS pathway as illustrated in Fig. 8(b). As shown in Fig. 8(c-f), the expression of the anti-ROS enzymatic machinery in the proximal (duodenum) $v$. distal (ileum) bowel correlated with the observed distribution of NEC after the 
Proximal intestine (duodenum)

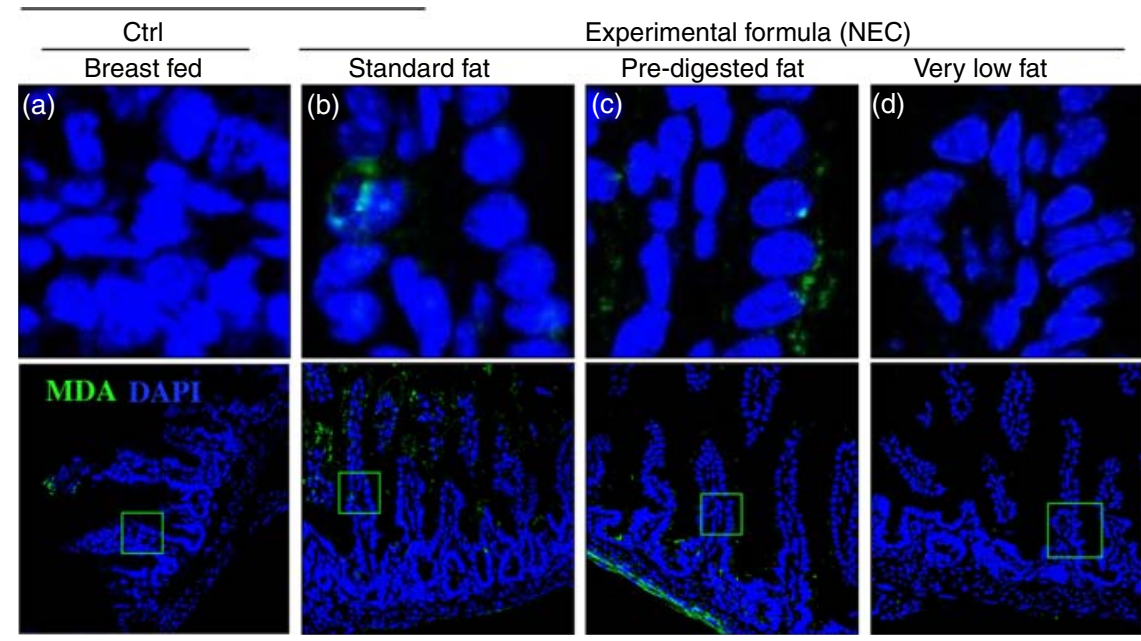

Distal intestine (ileum)
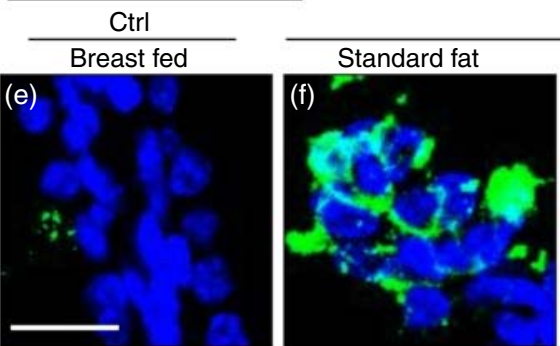

Experimental formula (NEC)


Fig. 6. The effect of fat composition on the accumulation of oxidised lipids in ileal enterocytes of mice induced to develop necrotising enterocolitis (NEC). (a-h) Immunofluorescence images of malondialdehyde (MDA, green) and 4',6-diamidino-2-phenylindole, dihydrochloride (DAPI) stained (nuclei, blue) from control and NEC mice proximal (duodenum (a-d)) and distal (ileum (e-h)) small intestine (10 $\mu$ m, cryo-sections) (Ctrl, control-breast-fed or experimental NEC treatments with hypoxia and formula feeding). Scale bar $=10 \mu \mathrm{m}$.

accumulation of fat. Specifically, the expression levels of the ROS donor NADPH oxidase 2 and the $\mathrm{H}_{2} \mathrm{O}_{2}$ donor superoxide dismutase 2 were significantly increased in the ileum of NEC mice and decreased in the duodenum (Fig. 8(c)), whereas the ROS sensors and cytoprotective transcription factors Kelch-like ECH-associated protein 1 and Nrf2 were expressed at very low levels in the ileum as compared with the duodenum, suggesting the presence of an antioxidant environment under physiological conditions of the duodenum. The gene expression data of Nrf2 were verified by immunostaining for Nrf2 in the duodenum (Fig. 8(g)i-ii) and ileum (Fig. 8(g)iii-iv), revealing low levels in the ileum. Furthermore, the mRNA levels of $N r f 2-$ dependent anti-oxidative enzymes $N A D(P) H$ quinone debydrogenase 1, thioredoxin reductase 1 and toxic peroxide detoxification enzymes intestinal glutathione peroxidase 2, aldo-keto reductase family 1 , member $B 7$, were significantly lower in the ileum compared with the duodenum and further reduced in the ileum of NEC mice (Fig. 8(e) and (f)). These data suggest that the proximal intestine exists in an antioxidant environment, which contributes to reduced inflammation after the administration of lipid, as compared with the pro-inflammatory, pro-oxidant environment in the distal intestine, which predisposes to intestinal injury and NEC.

\section{Discussion}

This study provides evidence that the composition of fat in infant formula has a significant effect on the severity of NEC, and further that the administration of a novel formula containing 'PDF', composed of soyabean oil NEFA, monoacylglycerol palmitate and soya lecithin, can reduce NEC severity. In seeking to understand the mechanisms involved, we focused on the observation that in the premature gastrointestinal tract the expression of lipases is relatively low, resulting in the delivery of undigested lipid constituents to the distal ileum, where their intracellular accumulation can lead to the generation of ROS, 
(a)

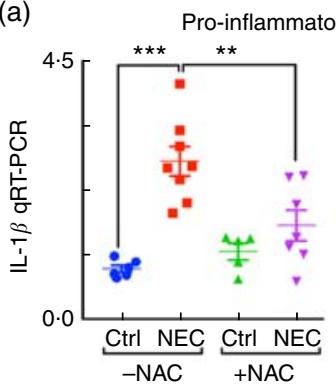

(b)

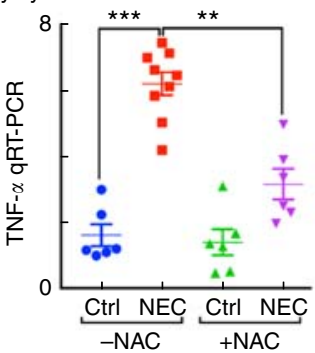

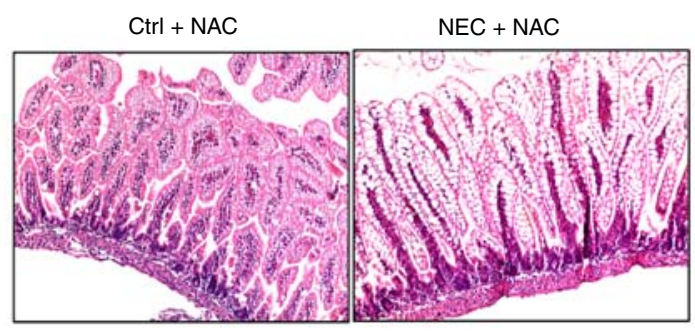

(c) NEC severity

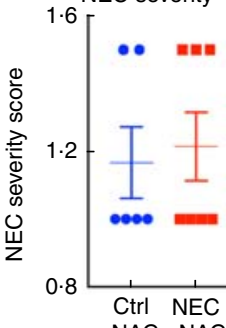

\section{Experimental NEC}

$-\mathrm{NAC}$
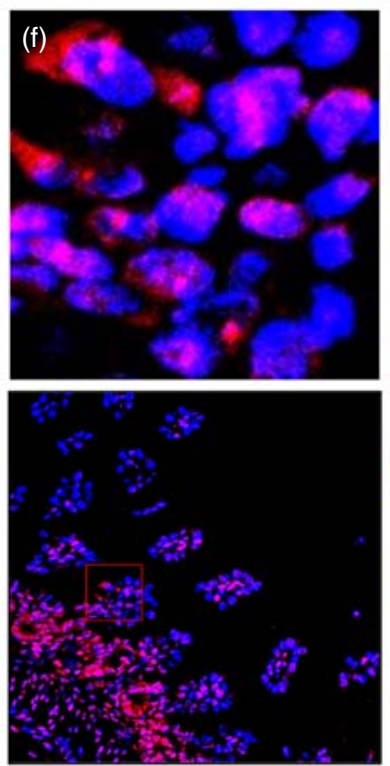

(j)
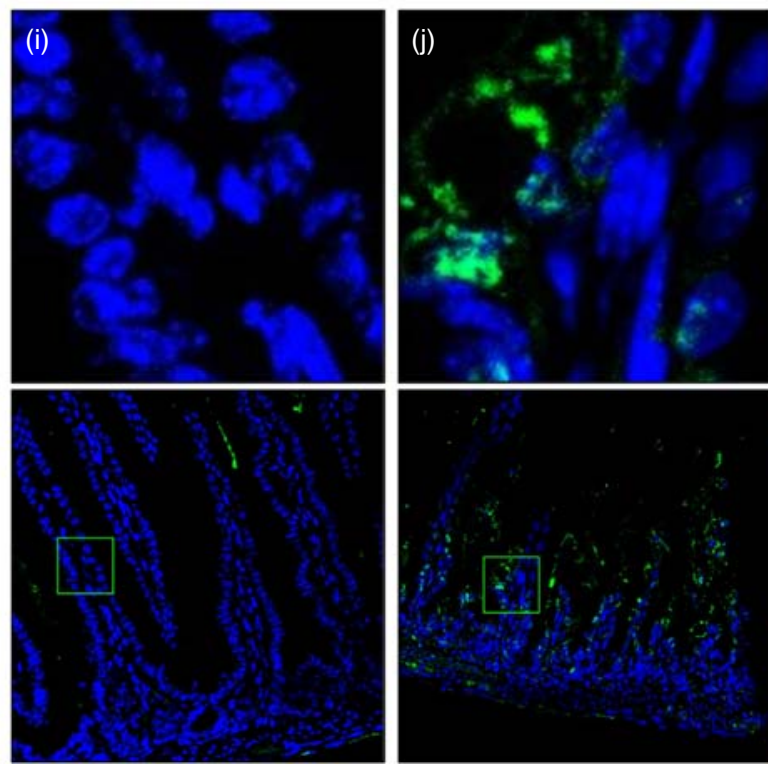
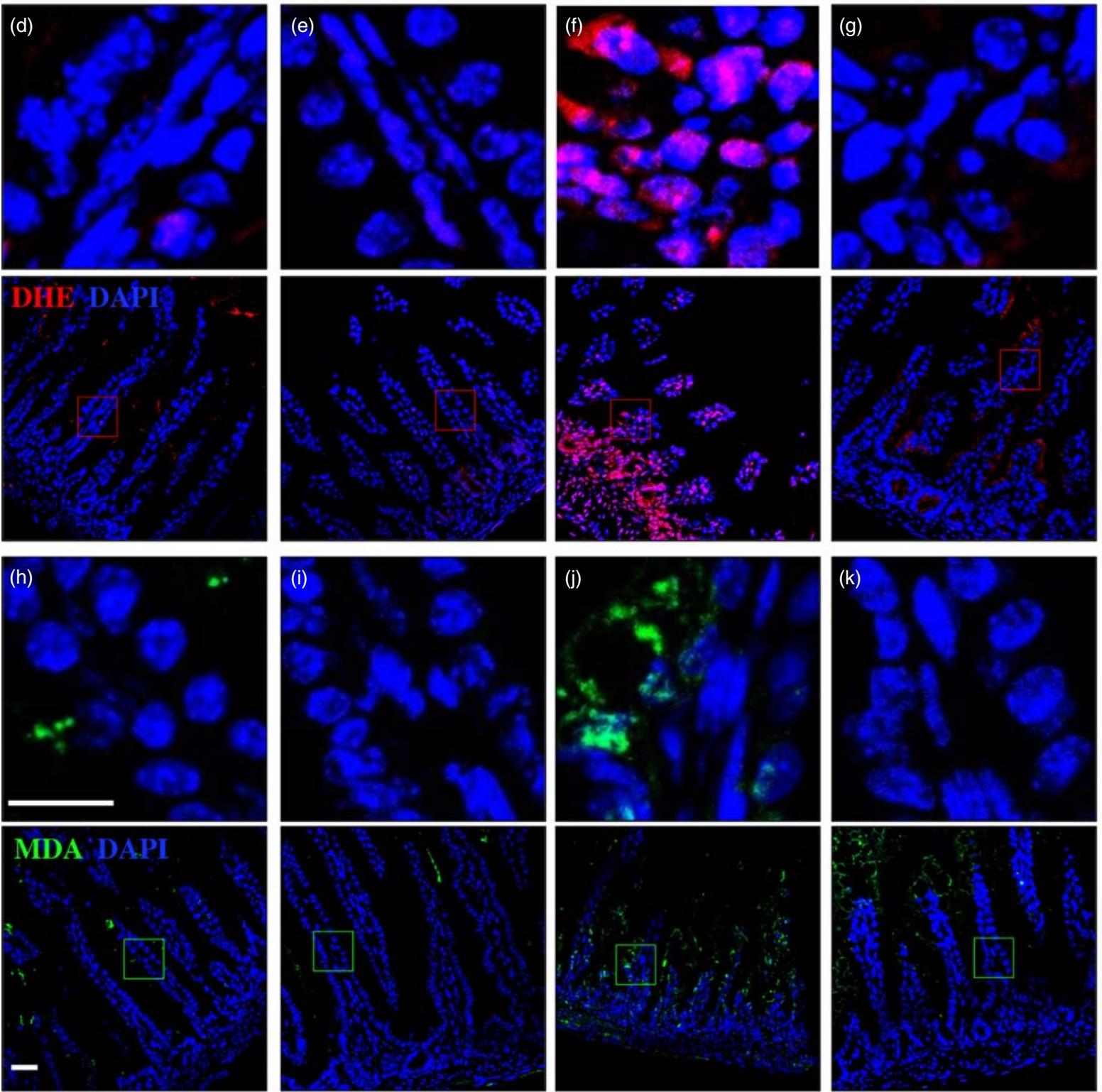

Fig. 7. Administration of the reactive oxygen species (ROS) scavenger $\mathrm{N}$-acetylcysteine (NAC) prevents ROS generation and necrotising enterocolitis (NEC) development. (a) Quantitative real-time PCR (qRT-PCR) of pro-inflammatory cytokines. (b) Haematoxylin-eosin (H\&E)-stained images. (c) NEC severity score. (d-g) Immunofluorescence images of dihydroxyethidium (DHE, red) and 4',6-diamidino-2-phenylindole, dihydrochloride (DAPI) staining (nuclei, blue). (h-k) Immunofluorescence images of malondialdehyde (MDA, green) and DAPI staining (nuclei, blue) from the distal intestine (ileum) of control and NEC mice (Ctrl, control-breast-fed or experimental NEC treatments with and without NAC supplementation). ${ }^{\star \star} P<0.01,{ }^{\star \star *} P<0.001$ by Student's $t$ test when comparisons of two groups were made, and by ANOVA for multiple comparisons; each dot represents data from an individual mouse, scale bar $=10 \mu \mathrm{m}$. 
(a)

Pro-inflammatory cytokines
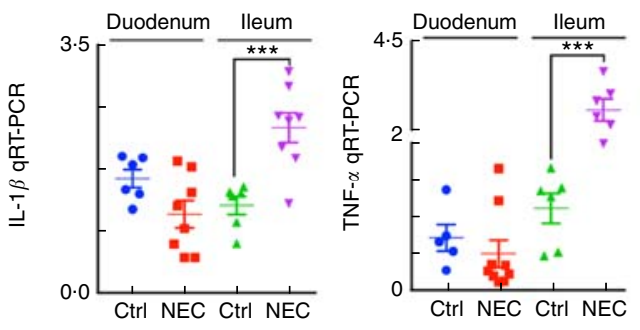

(c)

ROS donor


(e)

\section{Anti-oxidative response}
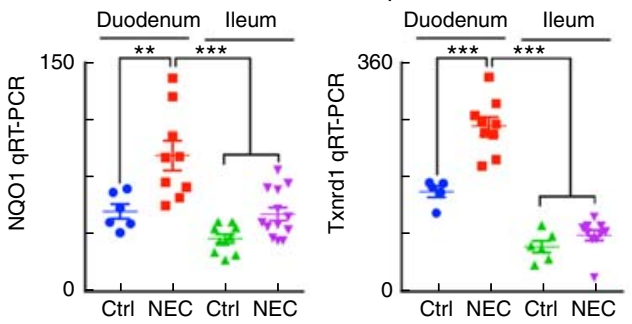

Proximal intestine (duodenum)

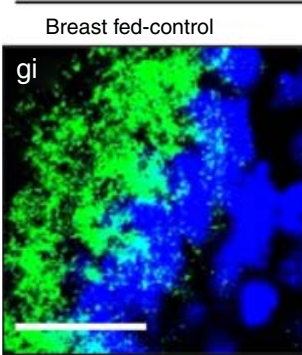

Experimental NEC
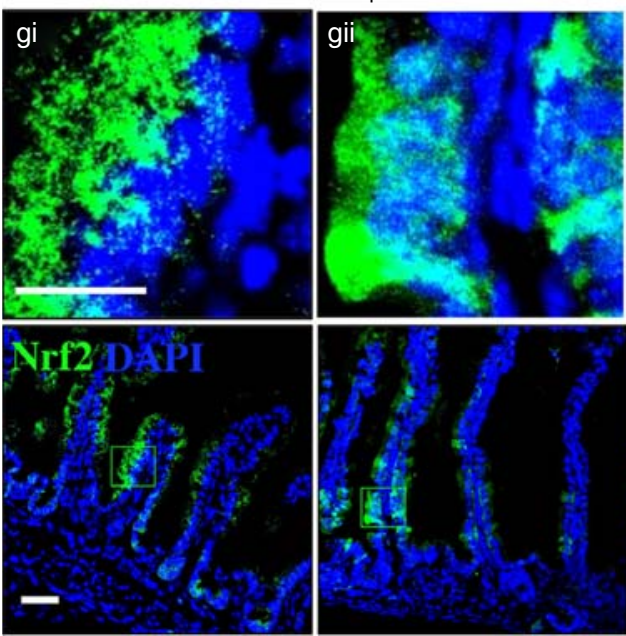

(b)

Nrf2/ROS pathway

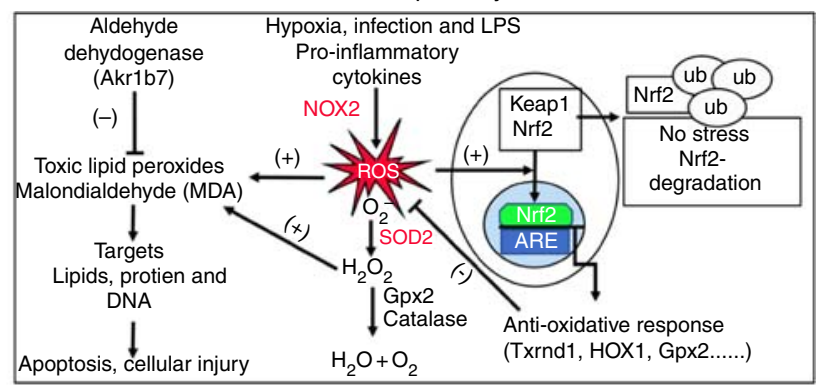

(d)

ROS sensor

(f)

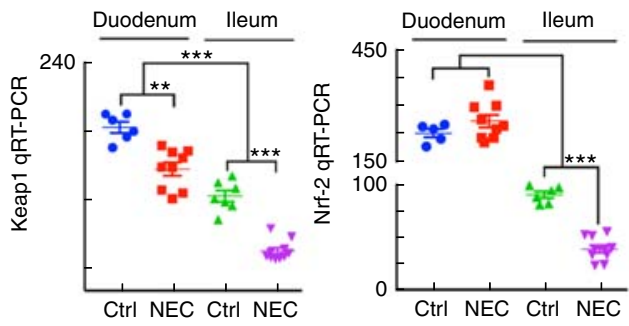

Toxic peroxide detoxification



Distal intestine (ileum)

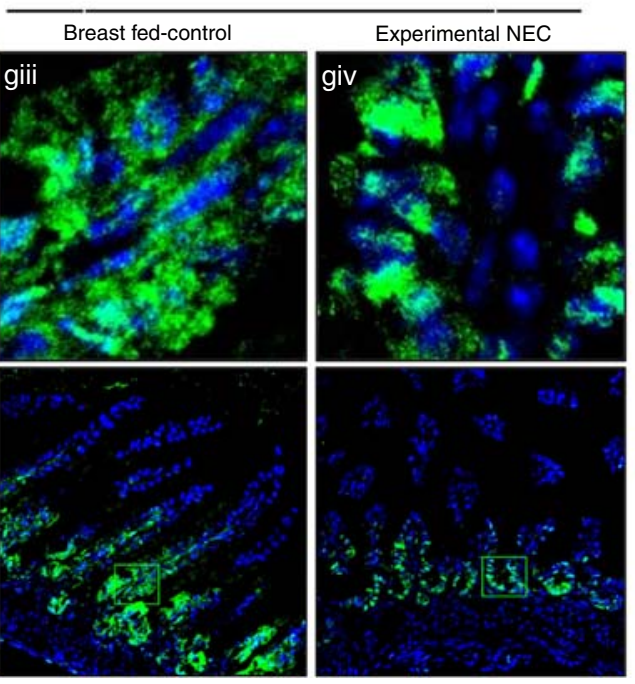

Fig. 8. The antioxidative environment of the proximal small intestine $v$. the distal small intestine correlates with the location of necrotising enterocolitis (NEC). (a) Quantitative real-time PCR (qRT-PCR) of pro-inflammatory cytokines IL-1 $\beta$ and TNF- $a$. (b) Schematic of nuclear factor erythroid 2 p45-related factor (Nrf2)/reactive oxygen species (ROS) oxidative injury pathway in the intestinal epithelium. (c) qRT-PCR of ROS donor enzymes NADPH oxidase (Nox2) and superoxide dismutase (SOD2, mitochondrial). (d) qRTPR of ROS sensor kelch-like ECH-associated protein 1 (Keap1) and Nrf2. (e) qRT-PCR of antioxidants NADPH quinone dehydrogenase 1 (NQO1) and thioredoxin reductase 1 (Txnrd1). (f) qRT-PCR of peroxide detoxifier glutathione peroxidase 2 (Gpx2, intestinal) and aldo-keto reductase family 1, member B7 (Akr1b7). (g) Immunofluorescence images of Nrf2 showing cytoplasmic and nuclear translocation (Nrf2, green) and 4',6-diamidino-2-phenylindole, dihydrochloride (DAPI) (nuclei, blue) in control and NEC mice (Ctrl, control-breast-fed or experimental NEC). ${ }^{\star \star} P<0.01$, ${ }^{\star \star \star} P<0.001$ by Student's $t$ test when comparisons of two groups were made, and by ANOVA for multiple comparisons; each dot represents data from an individual mouse. Akr1b7 (aldo-keto reductase family 1 , member B7). Scale bar $=10 \mu \mathrm{m}$. 
oxidative stress and the inflammation that characterises NEC. The oral administration of the broad-acting antioxidant NAC reversed the accumulation of ROS and limited the degree of NEC and NEC-induced injury in mice, providing support for the role of ROS in NEC. We further determined that the proximal bowel has a greater expression of antioxidant genes as compared with the distal bowel, which may partly explain the greater susceptibility of the distal bowel to NEC as compared with the proximal bowel, while also explaining how the intracellular accumulation of lipid may have variable effects in different parts of the bowel. Taken together, these findings shed light on how the rational development of infant formulas through the provision of a 'PDF' substrate may offer preventative strategy for infants at risk for the development of NEC.

The modified formula containing a novel 'PDF' system that was used in the present study merits additional discussion. NEFA included in the 'PDF' are normal metabolic breakdown products of TAG after lipase action in the gastrointestinal tract. These NEFA are packaged into micelles that are absorbed readily by diffusion in the proximal intestine into epithelial cells where they are then converted back into TAG with monoacylglycerol and subsequently transported out to the rest of the body as chylomicrons via the lymphatics. The Ca salt of NEFA present in the 'PDF' formula could mimic metabolites of the normal digestive process and be readily absorbed in the proximal intestine. The 'PDF' formula is different from a typical medium-chain TAG (MCT)-rich infant formula, as the fat content used is a mixture of soyabean NEFA (17.5\%), monoacylglycerol palmitate (20\%) and phospholipid lecithin (10.3\%) to bring the fatty acid profile closer to that of human milk. Soyabean NEFA are produced from naturally occurring soyabean oil (fatty acid in TAG form), which is highly processed by hydrolysis and distillation in order to separate glycerol from NEFA to achieve their final composition. In contrast, 'standard fat'-containing formula contains non-hydrolysed oils (soyabean oil, HOSO and coconut oil) in $100 \%$ TAG form. This oil composition thus requires endogenous lipase activity for successful digestion and absorption, without which the accumulation of undigested TAG containing especially unsaturated fatty acids, for example, linoleic and $\alpha$-linolenic, leads to the accumulation of ROS and cellular injury. It is noteworthy that in the current model of experimental NEC mice displayed significantly reduced pancreatic lipase activity as compared with breast-fed controls, perhaps as a result of hypoxia during the highly vulnerable window of the 1st week or so of life. This lack of lipase partly explains the reduced ability of these mice to undergo fat digestion, leading to NEC. Importantly, these cytotoxic effects could be reversed by the addition of the PDF formula, thus linking the presence of undigested fat with NEC development.

With respect to understanding further how breast milk protects against NEC, it should be mentioned that the fat-digestive enzyme carboxyl ester lipase (CEL, also called bile-saltdependent lipase, BSDL) is not only released by the pancreas, but is also present at very high levels in the breast milk of several species including mouse and human ${ }^{(17,18,36,37)}$, where it plays a critical key role in digestion and absorption of milk fat, which itself exists as a TAG form. Several investigators have demonstrated that in preterm infants the pancreas is unable to secrete sufficient lipases for TAG fat digestion owing to enzyme insufficiency ${ }^{(13,38,39)}$, and indeed the data in Fig. 4(a) indicating the ontogeny of CEL secretion are consistent with this concept. Although mice that were induced to develop NEC were unable to digest the TAG fat completely when fed 'standard formula', the breast-fed pups were able to digest the milk fat (100\% TAG fat), likely owing to the presence of the fat-digestive enzymes that are present in the breast milk, accounting in part for why undigested fat did not accumulate in the lumen of breast-fed mice. Casper et $a l .{ }^{(16)}$ have demonstrated that by supplementing recombinant human CEL in formula they facilitated fat digestion and absorption in preterm infants. The presence of lipases ${ }^{(36)}$ in the breast milk supports the rationale for the PDF approach, which bypasses the requirement for CEL for fat digestion and absorption.

The current study adds to a growing body of work regarding the role of fat composition of infant formula on the maintenance of infant health. Caplan et $a l^{(3)}$ have shown in experimental systems that the administration of a combination of long-chain PUFA, specifically ARA and DHA, can reduce intestinal inflammation and attenuate the degree of experimental NEC in a neonatal rat model, whereas DHA alone was unable to show any beneficial effect in terms of reducing NEC or TLR4 expression ${ }^{(40)}$. In these studies, the authors used formula supplemented with $34 \mathrm{mg} / 100 \mathrm{ml}$ ARA and $23 \mathrm{mg} / 100 \mathrm{ml}$ DHA. Although in the present study the formulas (standard, PDF) contain approximately the same amount of both ARA (approximately $32 \mathrm{mg} / 100 \mathrm{ml}$ ) and DHA (approximately $15 \mathrm{mg} / 100 \mathrm{ml}$ ) (Table 1), these two formulas showed different outcomes indicating that fat digestion and absorption in the current study may play an important role in NEC development. There are additional studies over the past several decades that have confirmed the various health benefits of PUFA, whose effects include improved absorption, enhanced membrane integrity, anti-inflammatory effects and salutary effects on cell function $^{(3,41,42)}$.

We readily acknowledge that the present study has several limitations that may prevent its translation to the human population. First, we have shown data from a mouse model, which although shares many similarities with the human NEC, is limited by the short life span of the mouse compared to the human, and the fact that the mouse model cannot be subjected to surgical intervention or ostomy creation for further translatability of the current findings. Further, we acknowledge that there may be additional subtle differences in the various formulas that we have tested here, although the major differences lie in the type of fat components. Finally, we recognise that one of the key and perhaps underappreciated effects of the fat component in the diet on the premature infant will be on the microbiome, which itself can have major effects on the propensity for NEC development. Additional studies will be required to determine in greater detail the degree to which the PDF formula can affect the microbiome, and the effects to which changes in the microbiome can lead to NEC.

\section{Acknowledgements}

D. J. H. is supported by grants (R01GM078238 and R01DK083752) from the National Institutes of Health and this 
research was funded in part by a Sponsored Research Grant from Abbott Nutrition. M. G. is supported by grants (K08DK101608 and R03DK111473) from the National Institutes of Health, March of Dimes Foundation (grant no. 5-FY17-79), the Children's Discovery Institute of Washington University and St Louis Children's Hospital. There was no role by Abbott Nutrition in the design of the study, collation and analysis of data or decision to publish

C. P. S., W. B. F., M. G., M. F., T. D., C. L. and D. J. H. designed and/or performed experiments, analysed results, wrote and edited the manuscript. H. J., Y. Y., P. L., T. P. and J. A. O. performed experiments and analysed data.

None of the authors has any conflicts of interest to declare.

The amount of all nutrients used in animal studies could reasonably be expected to be achieved in the human population as they are derived from ready to feed infant formulas. There are no in vitro studies, so the statement as requested by the British Journal of Nutrition that the 'molecular form of the nutrient or nutrients used in in vitro studies is the same as that which the cell type in which the test was performed would encounter in vivo' does not apply. This work does not involve any probiotics.

\section{References}

1. Nino DF, Sodhi CP \& Hackam DJ (2016) Necrotizing enterocolitis: new insights into pathogenesis and mechanisms. Nat Rev Gastroenterol Hepatol 13, 590-600.

2. Neu J \& Walker WA (2011) Necrotizing enterocolitis. N Engl J Med 364, 255-264.

3. Caplan MS, Russell T, Xiao Y, et al. (2001) Effect of polyunsaturated fatty acid (PUFA) supplementation on intestinal inflammation and necrotizing enterocolitis (NEC) in a neonatal rat model. Pediatr Res 49, 647-652.

4. Lu J, Jilling T, Li D, et al. (2007) Polyunsaturated fatty acid supplementation alters proinflammatory gene expression and reduces the incidence of necrotizing enterocolitis in a neonatal rat model. Pediatr Res 61, 427-432.

5. Egan CE, Sodhi CP, Good M, et al. (2016) Toll-like receptor 4-mediated lymphocyte influx induces neonatal necrotizing enterocolitis. J Clin Invest 126, 495-508.

6. Soliman A, Michelsen KS, Karahashi H, et al. (2010) Plateletactivating factor induces TLR4 expression in intestinal epithelial cells: implication for the pathogenesis of necrotizing enterocolitis. PLOS ONE 5, e15044.

7. Yin Y, Liu F, Li Y, et al. (2016) mRNA expression of TLR4, TLR9 and NF-kappaB in a neonatal murine model of necrotizing enterocolitis. Mol Med Rep 14, 1953-1956.

8. Zhou Y, Li Y, Zhou B, et al. (2017) Inflammation and apoptosis: dual mediator role for toll-like receptor 4 in the development of necrotizing enterocolitis. Inflamm Bowel Dis 23, 44-56.

9. Innis SM (2011) Dietary triacylglycerol structure and its role in infant nutrition. Adv Nutr 2, 275-283.

10. Armand M, Hamosh M, Mehta NR, et al. (1996) Effect of human milk or formula on gastric function and fat digestion in the premature infant. Pediatr Res 40, 429-437.

11. Martin CR, Cheesman A, Brown J, et al. (2016) Factors determining optimal fatty acid absorption in preterm infants. J Pediatr Gastroenterol Nutr 62, 130-136.

12. Black DD (1992) Effect of intestinal chylomicron secretory blockade on apolipoprotein synthesis in the newborn piglet. Biochem J 283, 81-85.
13. Lindquist $S$ \& Hernell $O$ (2010) Lipid digestion and absorption in early life: an update. Curr Opin Clin Nutr Metab Care 13, 314-320.

14. Lebenthal E \& Lee PC (1980) Development of functional responses in human exocrine pancreas. Pediatrics 66, 556560.

15. Howles PN, Stemmerman GN, Fenoglio-Preiser CM, et al. (1999) Carboxyl ester lipase activity in milk prevents fatderived intestinal injury in neonatal mice. Am J Physiol 277, G653-G661.

16. Casper C, Carnielli VP, Hascoet JM, et al. (2014) rhBSSL improves growth and LCPUFA absorption in preterm infants fed formula or pasteurized breast milk. J Pediatr Gastroenterol Nutr 59, 61-69.

17. Ellis LA \& Hamosh M (1992) Bile salt stimulated lipase: comparative studies in ferret milk and lactating mammary gland. Lipids 27, 917-922.

18. Freed LM, York CM, Hamosh M, et al. (1986) Bile saltstimulated lipase in non-primate milk: longitudinal variation and lipase characteristics in cat and dog milk. Biochim Biophys Acta 878, 209-215.

19. Badwey JA, Curnutte JT, Robinson JM, et al. (1984) Effects of free fatty acids on release of superoxide and on change of shape by human neutrophils. Reversibility by albumin. J Biol Chem 259, 7870-7877.

20. Penn AH, Altshuler AE, Small JW, et al. (2014) Effect of digestion and storage of human milk on free fatty acid concentration and cytotoxicity. J Pediatr Gastroenterol Nutr 59, 365-373.

21. Kilkenny C, Browne WJ, Cuthill IC, et al. (2010) Improving bioscience research reporting: the ARRIVE guidelines for reporting animal research. PLOS Biol 8, e1000412.

22. Good M, Sodhi CP, Egan CE, et al. (2015) Breast milk protects against the development of necrotizing enterocolitis through inhibition of toll-like receptor 4 in the intestinal epithelium via activation of the epidermal growth factor receptor. Mucosal Immunol 8, 1166-1179.

23. Good M, Sodhi CP \& Hackam DJ (2014) Evidence-based feeding strategies before and after the development of necrotizing enterocolitis. Expert Rev Clin Immunol 10, 875-884.

24. Sodhi CP, Jia H, Yamaguchi Y, et al. (2015) Intestinal epithelial TLR- 4 activation is required for the development of acute lung injury after trauma/hemorrhagic shock via the release of HMGB1 from the gut. J Immunol 194, 4931-4939.

25. Hoshiba J (2004) Method for hand-feeding mouse pups with nursing bottles. Contemp Top Lab Anim Sci 43, 50-53.

26. Good M, Sodhi CP, Yamaguchi Y, et al. (2016) The human milk oligosaccharide 2'-fucosyllactose attenuates the severity of experimental necrotising enterocolitis by enhancing mesenteric perfusion in the neonatal intestine. Br J Nutr 116, $1175-1187$

27. Afrazi A, Branca MF, Sodhi CP, et al. (2014) Toll-like receptor 4-mediated endoplasmic reticulum stress in intestinal crypts induces necrotizing enterocolitis. J Biol Chem 289, 9584-9599.

28. Halpern MD, Weitkamp JH, Mount Patrick SK, et al. (2010) Apical sodium-dependent bile acid transporter upregulation is associated with necrotizing enterocolitis. Am J Physiol Gastrointest Liver Physiol 299, G623-G631.

29. Yazji I, Sodhi CP, Lee EK, et al. (2013) Endothelial TLR4 activation impairs intestinal microcirculatory perfusion in necrotizing enterocolitis via eNOS-NO-nitrite signaling. Proc Natl Acad Sci U S A 110, 9451-9456.

30. Livak KJ \& Schmittgen TD (2001) Analysis of relative gene expression data using real-time quantitative PCR and the 2 (-Delta Delta C(T)) method. Methods 25, 402-408. 
31. Zhang Y, Chen SY, Hsu T, et al. (2002) Immunohistochemical detection of malondialdehyde-DNA adducts in human oral mucosa cells. Carcinogenesis 23, 207-211.

32. Yajima M, Kanno T \& Yajima T (2006) A chemically derived milk substitute that is compatible with mouse milk for artificial rearing of mouse pups. Exp Anim 55, 391-397.

33. Sodhi C, Richardson W, Gribar S, et al. (2008) The development of animal models for the study of necrotizing enterocolitis. Dis Model Mech 1, 94-98.

34. Pryor WA \& Stanley JP (1975) Letter: A suggested mechanism for the production of malonaldehyde during the autoxidation of polyunsaturated fatty acids. Nonenzymatic production of prostaglandin endoperoxides during autoxidation. J Org Chem 40, 3615-3617.

35. Yin G, Wang Y, Cen XM, et al. (2015) Lipid peroxidationmediated inflammation promotes cell apoptosis through activation of NF-kappaB pathway in rheumatoid arthritis synovial cells. Mediators Inflamm 2015, 460310.

36. Olivecrona T \& Hernell O (1976) Human milk lipases and their possible role in fat digestion. Padiatr Padol 11, 600-604.
37. Miller R \& Lowe ME (2008) Carboxyl ester lipase from either mother's milk or the pancreas is required for efficient dietary triglyceride digestion in suckling mice. $J$ Nutr 138, 927-930.

38. Brady MS, Rickard KA, Fitzgerald JF, et al. (1986) Specialized formulas and feedings for infants with malabsorption or formula intolerance. J Am Diet Assoc 86, 191-200.

39. Nevin-Folino NL, Loughead JL \& Loughead MK (2001) Enhanced-calorie formulas: considerations and options. NeOnatal Netw 20, 7-15.

40. Diehl-Jones WL, Askin DF \& Friel JK (2007) Microlipidinduced oxidative stress in human breastmilk: in vitro effects on intestinal epithelial cells. Breastfeed Med 2, 209-218.

41. Kapoor V, Glover R \& Malviya MN (2015) Alternative lipid emulsions versus pure soy oil based lipid emulsions for parenterally fed preterm infants. Cochrane Database Syst Rev, issue 12, CD009172.

42. Vegge A, Thymann T, Lauritzen L, et al. (2015) Parenteral lipids and partial enteral nutrition affect hepatic lipid composition but have limited short term effects on formulainduced necrotizing enterocolitis in preterm piglets. Clin Nutr 34, 219-228. 